\title{
The surface-tension-driven evolution of a two-dimensional annular viscous tube
}

\author{
By I. M. GRIFFITHS A D P. D. HOWELL \\ Mathematical Institute, 24-29 St Giles', Oxford, OX1 3LB, UK
}

(Received 20 February 2007 and in revised form 6 August 2007)

We consider the evolution of an annular two-dimensional region occupied by viscous fluid driven by surface tension and applied pressure at the free surfaces. We assume that the thickness of the domain is small compared with its circumference so that it may be described as a thin viscous sheet whose ends are joined to form a closed loop. Analytical and numerical solutions of the resulting model are obtained and we show that it is well posed whether run forwards or backwards in time. This enables us to determine, in many cases explicitly, which initial shapes will evolve into a desired final shape. We also show how the application of an internal pressure may be used to control the evolution.

This work is motivated by the production of non-axisymmetric capillary tubing via the Vello process. Molten glass is fed through a die and drawn off vertically, while the shape of the cross-section evolves under surface tension and any applied pressure as it flows downstream. Here the goal is to determine the die shape required to achieve a given desired final shape, typically square or rectangular. We conclude by discussing the role of our two-dimensional model in describing the three-dimensional tube-drawing process.

\section{Introduction}

This study is motivated by the industrial manufacture of glass capillary tubing with a specified cross-sectional shape. In particular, an interest has developed in the production of tubing with a square or rectangular cross-section. The tubes may be used, for example, to make medicine bottles (Pfaender 1996), or square cross-sectional optical fibres (Wu et al. 1998, 2000).

Capillary tubing is typically manufactured by either the Danner drawing process or the Vello method. The Danner process is used to produce regular cylindrical tubing, while tubing with cross-sectional shapes other than circular are produced using the Vello method (Uhlmann \& Kreidl 1984), as illustrated in figure 1. Molten glass is fed through a die and the glass tubing is drawn off vertically, before being cut to rough length by a cutter. The profile of the cross-section varies downstream of the die in response to surface tension, and additional control may be achieved by applying an internal pressure (Uhlmann \& Kreidl 1984). The ultimate aim is to solve the inverse problem of determining the die shape required to achieve a given final (e.g. square) cross-section.

As a first step towards understanding the three-dimensional process, in this paper we consider an interesting two-dimensional analogue. We study Stokes flow in a fluid with constant viscosity which occupies a two-dimensional annular domain, driven by surface tension and applied pressures on the free boundaries. We are particularly interested in the inverse time evolution of this problem to determine the initial shape required to generate, for example, a square profile at a later time. To tackle this difficult free-boundary problem, we suppose the domain thickness is small compared with its circumference, a condition 
which is satisfied in practice by any cross-section of tubing in the three-dimensional tubedrawing problem. This allows us to describe the set-up as a thin two-dimensional viscous sheet or viscida (Buckmaster, Nachman \& Ting 1975; Buckmaster \& Nachman 1978), whose ends are joined to form a closed loop.

We begin in $\S 2$ by defining our curvilinear coordinate system and stating the governing equations and boundary conditions. By integrating across the viscida, we obtain three global equations representing net force and torque balances. Closure relations for these are obtained in $\S 3$ by non-dimensionalizing the equations and taking an asymptotic limit as the inverse aspect ratio of the viscida tends to zero. The resulting leading-order model comprises two evolution equations for the thickness and the centre-line of the viscida. These equations are simplified further by assuming that the viscida initially has uniform thickness and is rotationally symmetric; both are true for shapes of interest to capillary tubing manufacturers.

In $\S 4$ we find that when no pressure is applied to the sheet, the model may be solved exactly; when the pressure is non-zero, it is easily solved numerically. Surprisingly, the model is well posed whether run forwards or backwards in time, and we can thus solve the inverse problem of finding an initial shape that will evolve into some given final shape under surface tension. We find that the application of an internal pressure can indeed afford significant control over the shape evolution. In particular, it appears that a suitable negative pressure can almost fix the shape of the viscida, and this motivates us in $\S 5$ to examine the possibility of steady shapes that are preserved exactly through a balance between surface tension and applied pressure. We discover that such shapes do exist and that some of them are tantalizingly close to the polygonal shapes that we wish to produce. Furthermore, we go on to show in $\S 6$ that the evolution of approximately polygonal shapes can be well described using a simple weakly nonlinear theory that admits exact solution for regimes of practical interest.

We conclude by discussing how the analysis of this two-dimensional Stokes flow problem may be extended to describe the three-dimensional temperature-dependent tube-drawing problem, the details of which will be reported in a subsequent paper.

\section{Governing equations and boundary conditions}

\subsection{Typical parameter values}

Capillary tubing is usually produced using glass consisting of approximately $75 \%$ silica, $15 \%$ soda and 10\% lime, and typical parameter values are (Graham 1987; Sivko 1976)

$$
\begin{aligned}
& \text { surface tension: } \quad \gamma \sim 0.3 \mathrm{~N} \mathrm{~m}^{-1}, \\
& \text { density: } \quad \rho \sim 2500 \mathrm{~kg} \mathrm{~m}^{-3} \text {, } \\
& \text { viscosity: } \quad \mu \sim 10^{2}-10^{8} \mathrm{~N} \mathrm{~s} \mathrm{~m}^{-2} \text {, } \\
& \text { radius: } \quad r \sim 20 \mathrm{~mm} \text {, } \\
& \text { thickness: } \quad h \sim 0.8 \mathrm{~mm} \text {. }
\end{aligned}
$$

With the circumference of the cross-section denoted by $L \sim 2 \pi r$, a typical inverse aspect ratio, or slenderness parameter, is given by

$$
\epsilon=\frac{h}{L} \sim 0.006
$$




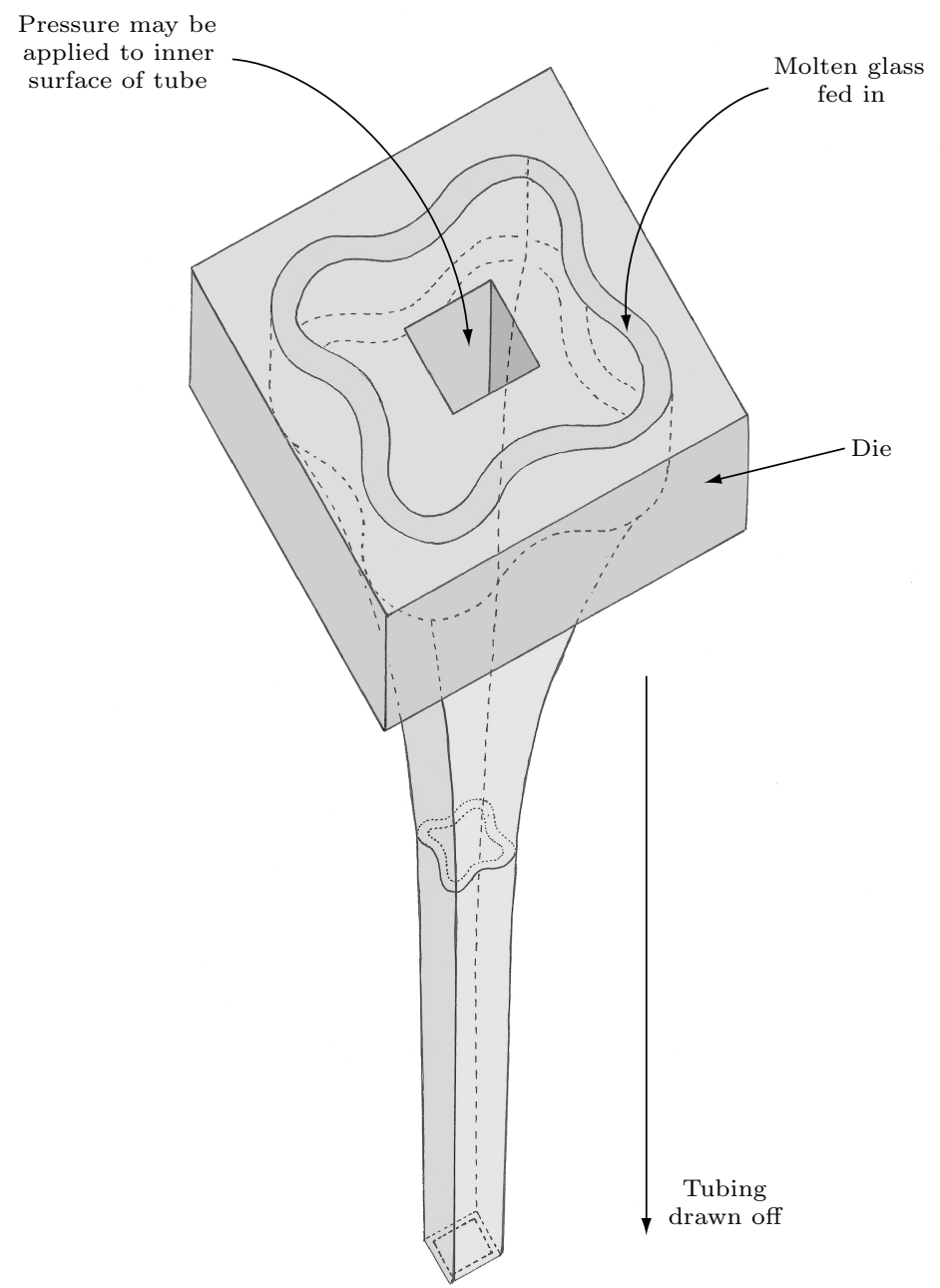

FIGURE 1. Schematic diagram of the Vello process for the construction of square capillary tubing.

and with a velocity scale of $U=\gamma / \epsilon \mu$, the Reynolds number is given by

$$
R e=\frac{\rho \gamma L}{\epsilon \mu^{2}} \sim 10^{-12}-10^{0} .
$$

Note that for the fabrication of optical fibres, values for the radius and thickness vary significantly (Fitt et al. 2001, 2002).

We are thus motivated to model the tube wall as a two-dimensional thin viscida driven by surface tension. We may also neglect inertia and hence use the Stokes equations, except under extreme conditions of very low viscosity and thin walls. Equations governing the evolution of a viscida were previously derived by Buckmaster et al. (1975) and Buckmaster \& Nachman (1978). We follow a more systematic approach based on asymptotic analysis of the governing Stokes equations and boundary conditions, without using any 
ad hoc kinematic assumptions. At the same time, we incorporate an applied pressure drop across the viscida. However, we omit many of the straightforward but messy details.

\subsection{Geometry of the viscida}

We choose a coordinate system which is fixed in the evolving viscida, denoting the position of its centre-line by $\boldsymbol{r}=\boldsymbol{r}_{\boldsymbol{c}}(s, t)$, where $s$ is arc-length along the centre-line and $t$ is time. We let $n$ denote distance in the normal direction from the centre-line and $h(s, t)$ be the thickness of the sheet, so that the inner and outer surfaces of the sheet are given by $n= \pm h / 2$ respectively. We also denote by $\theta(s, t), \theta^{+}(s, t)$ and $\theta^{-}(s, t)$ the angles made in the anticlockwise sense between a chosen fixed horizontal axis and the centre-line, the inner surface and the outer surface respectively, as illustrated in figure 2.

We define the curvature of the centre-line $\kappa$, and the curvatures of the inner and outer surfaces $\kappa^{ \pm}$by

$$
\kappa=\frac{\partial \theta}{\partial s}, \quad \kappa^{ \pm}=\frac{\partial \theta^{ \pm}}{\partial s^{ \pm}}
$$

where $s^{ \pm}$represent arc-length along the inner and outer surfaces respectively. The unit tangent and normal to the centre-line in the directions of increasing $s$ and $n$ are denoted by

$$
\boldsymbol{t}(s, t)=\left(\begin{array}{c}
\cos \theta(s, t) \\
\sin \theta(s, t)
\end{array}\right), \quad \boldsymbol{n}(s, t)=\left(\begin{array}{c}
-\sin \theta(s, t) \\
\cos \theta(s, t)
\end{array}\right)
$$

and are related by the Serret-Frenet formulae (Kreyszig 1959),

$$
\frac{\partial \boldsymbol{t}}{\partial s}=\kappa \boldsymbol{n}, \quad \frac{\partial \boldsymbol{n}}{\partial s}=-\kappa \boldsymbol{t} .
$$

The tangent and normal to the inner and outer free surfaces are given by

$$
\boldsymbol{t}^{ \pm}=\frac{\left(1 \mp \frac{1}{2} h \kappa\right) \boldsymbol{t} \pm \frac{1}{2} h_{s} \boldsymbol{n}}{\sqrt{\frac{1}{4} h_{s}^{2}+\left(1 \mp \frac{1}{2} h \kappa\right)^{2}}}, \quad \quad \boldsymbol{n}^{ \pm}=\frac{\mp \frac{1}{2} h_{s} \boldsymbol{t}+\left(1 \mp \frac{1}{2} \kappa h\right) \boldsymbol{n}}{\sqrt{\frac{1}{4} h_{s}^{2}+\left(1 \mp \frac{1}{2} h \kappa\right)^{2}}}
$$

We let $v_{s}(s, t)$ and $v_{n}(s, t)$ be the velocity components of the centre-line in the tangential and normal directions, so that

$$
\frac{\partial \boldsymbol{r}_{\boldsymbol{c}}}{\partial t}=v_{s} \boldsymbol{t}+v_{n} \boldsymbol{n}
$$

Cross-differentiation reveals the identities

$$
\frac{\partial v_{s}}{\partial s}=\kappa v_{n}, \quad \frac{\partial \theta}{\partial t}=\kappa v_{s}+\frac{\partial v_{n}}{\partial s} .
$$

2.3. Governing equations and boundary conditions

The fluid flow in the viscida is governed by the Stokes equations, (Buckmaster et al., 1975; van de Fliert, Howell \& Ockendon, 1995)

$$
\begin{aligned}
\frac{\partial u_{s}}{\partial s}+\frac{\partial}{\partial n}\left(l u_{n}\right) & =0 \\
\frac{\partial \sigma_{s s}}{\partial s}+\frac{\partial}{\partial n}\left(l \sigma_{s n}\right)-\kappa \sigma_{s n} & =0 \\
\frac{\partial \sigma_{s n}}{\partial s}+\frac{\partial}{\partial n}\left(l \sigma_{n n}\right)+\kappa \sigma_{s s} & =0
\end{aligned}
$$




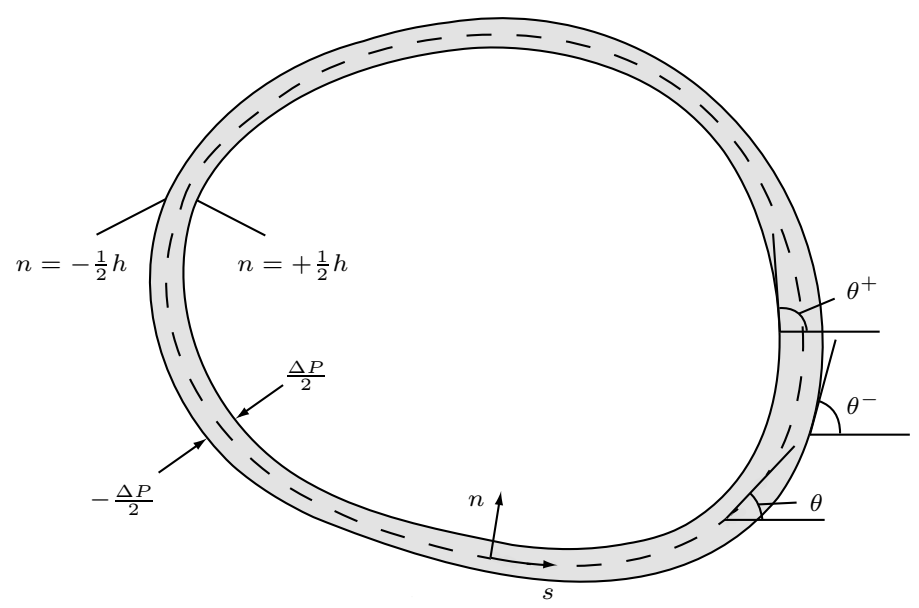

FiguRE 2. Schematic diagram of the coordinate system for the sheet.

neglecting any body forces. Here $l=1-\kappa n$ is the metric coefficient, $\boldsymbol{u}(s, n, t)=u_{s}(s, n, t) \boldsymbol{t}+$ $u_{n}(s, n, t) \boldsymbol{n}$ denotes the fluid velocity, and $\boldsymbol{\sigma}$ is the Newtonian stress tensor, with components

$$
\begin{aligned}
\sigma_{s s} & =-p+\frac{2 \mu}{l}\left(\frac{\partial u_{s}}{\partial s}+u_{n} \frac{\partial l}{\partial n}\right), \\
\sigma_{s n} & =\frac{\mu}{l}\left(l \frac{\partial u_{s}}{\partial n}-\frac{\partial l}{\partial n} u_{s}+\frac{\partial u_{n}}{\partial s}\right), \\
\sigma_{n n} & =-p+2 \mu \frac{\partial u_{n}}{\partial n}
\end{aligned}
$$

where $p$ is the fluid pressure and $\mu$ is its viscosity.

The kinematic condition may be written as (van de Fliert et al. 1995)

$$
u_{n}-v_{n}= \pm \frac{1}{2} \frac{\partial h}{\partial t} \pm \frac{1}{2 l} \frac{\partial h}{\partial s}\left[u_{s}-v_{s} \pm \frac{1}{2} h\left(\kappa v_{s}+\frac{\partial v_{n}}{\partial s}\right)\right]
$$

on $n= \pm h / 2$. The dynamic boundary conditions are

$$
\boldsymbol{\sigma} \cdot \boldsymbol{n}^{ \pm}= \pm \gamma \kappa^{ \pm} \boldsymbol{n}^{ \pm} \mp \frac{\Delta P}{2} \boldsymbol{n}^{ \pm}
$$

on $n= \pm h / 2$, where $\gamma$ is the surface tension, and $\pm \Delta P / 2$ represent the pressures inside and outside the viscida, so that $\Delta P$ is the net internal pressurization, which we assume to be spatially uniform.

The problem is closed by specifying the initial shape and thickness of the viscida and 
periodic boundary conditions on each end, namely

$$
\begin{aligned}
\boldsymbol{r}_{\boldsymbol{c}}(0, t) & =\boldsymbol{r}_{\boldsymbol{c}}(L, t), \\
\theta(0, t) & =0, \\
\theta(L, t) & =2 \pi, \\
\boldsymbol{u}(0, t) & =\boldsymbol{u}(L, t),
\end{aligned}
$$

where $L(t)$ is the sheet length, determined from conservation of mass by

$$
\int_{0}^{L(t)} h \mathrm{~d} s=\int_{0}^{L(0)} h(s, 0) \mathrm{d} s .
$$

Condition (2.18) ensures that the ends are joined, while (2.19) fixes the orientation of the profile, eliminating the arbitrary rigid-body rotation, and (2.20) ensures that the join is smooth. The final condition (2.21) states that the velocity field must be single-valued as the loop is traversed. However, there is still an arbitrary rigid-body translation which might be eliminated, for example, by setting $\boldsymbol{r}_{\boldsymbol{c}}(0, t) \equiv \mathbf{0}$, although we will find below that it is more convenient instead to fix the centre-of-mass of the viscida.

\subsection{Global force balances}

It is advantageous to use global force balances in the $s$ - and $n$-directions, obtained by integrating (2.11) and (2.12) over the thickness of the sheet, and a global torque balance obtained by multiplying (2.11) by $n$ before integrating over the thickness of the sheet. The resulting equations may be written as

$$
\begin{aligned}
\frac{\partial \mathcal{T}}{\partial s} & =\kappa \mathcal{F}, \\
\frac{\partial \mathcal{F}}{\partial s} & =-\kappa \mathcal{T}+\Delta P, \\
\frac{\partial \mathcal{M}}{\partial s} & =\mathcal{F}-\frac{\Delta P}{4} h \frac{\partial h}{\partial s},
\end{aligned}
$$

where

$$
\begin{aligned}
\mathcal{T} & =\int_{-h / 2}^{h / 2} \sigma_{s s} \mathrm{~d} n+\gamma \boldsymbol{t}^{+} \cdot \boldsymbol{t}+\gamma \boldsymbol{t}^{-} \cdot \boldsymbol{t} \\
& =\int_{h / 2}^{h / 2} \sigma_{s s} \mathrm{~d} n+\frac{\gamma\left(1-\frac{1}{2} h \kappa\right)}{\sqrt{\frac{1}{4} h_{s}^{2}+\left(1-\frac{1}{2} h \kappa\right)^{2}}}+\frac{\gamma\left(1+\frac{1}{2} h \kappa\right)}{\sqrt{\frac{1}{4} h_{s}^{2}+\left(1+\frac{1}{2} h \kappa\right)^{2}}} \\
\mathcal{F} & =\int_{-h / 2}^{h / 2} \sigma_{s n} \mathrm{~d} n+\gamma \boldsymbol{t}^{+} \cdot \boldsymbol{n}+\gamma \boldsymbol{t}^{-} \cdot \boldsymbol{n} \\
& =\int_{-h / 2}^{h / 2} \sigma_{s n} \mathrm{~d} n+\frac{\frac{1}{2} \gamma h_{s}}{\sqrt{\frac{1}{4} h_{s}^{2}+\left(1-\frac{1}{2} h \kappa\right)^{2}}}-\frac{\frac{1}{2} \gamma h_{s}}{\sqrt{\frac{1}{4} h_{s}^{2}+\left(1+\frac{1}{2} h \kappa\right)^{2}}} \\
\mathcal{M} & =\int_{-h / 2}^{h / 2} n \sigma_{s s} \mathrm{~d} n+\frac{1}{2} \gamma h \boldsymbol{t}^{+} \cdot \boldsymbol{t}-\frac{1}{2} \gamma h \boldsymbol{t}^{-} \cdot \boldsymbol{t} \\
& =\int_{-h / 2}^{h / 2} n \sigma_{s s} \mathrm{~d} n+\frac{\frac{1}{2} \gamma h\left(1-\frac{1}{2} \kappa h\right)}{\sqrt{\frac{1}{4} h_{s}^{2}+\left(1-\frac{1}{2} h \kappa\right)^{2}}}-\frac{\frac{1}{2} \gamma h\left(1+\frac{1}{2} \kappa h\right)}{\sqrt{\frac{1}{4} h_{s}^{2}+\left(1+\frac{1}{2} h \kappa\right)^{2}}}
\end{aligned}
$$

which represent respectively the total tension, shear force and bending moment in the viscida due to both viscous and surface-tension effects. 
Introduction of the total stresses in the $x$ - and $y$-directions, say $\mathcal{A}$ and $\mathcal{B}$, given by

$$
\mathcal{A}=\mathcal{T} \cos \theta-\mathcal{F} \sin \theta, \quad \mathcal{B}=\mathcal{T} \sin \theta+\mathcal{F} \cos \theta,
$$

allows us to write equations $(2.23)-(2.25)$ as

$$
\begin{aligned}
\frac{\partial \mathcal{A}}{\partial s} & =-\Delta P \sin \theta \\
\frac{\partial \mathcal{B}}{\partial s} & =\Delta P \cos \theta \\
\frac{\partial \mathcal{M}}{\partial s} & =-\mathcal{A} \sin \theta+\mathcal{B} \cos \theta-\frac{1}{4} \Delta P h \frac{\partial h}{\partial s},
\end{aligned}
$$

which represent global stress balances in the $x$ - and $y$-direction, and the global torque balance. We emphasize that no approximations have been employed thus far and hence (2.30)-(2.32) are exact. However, they are underdetermined, and it remains to derive an evolution equation for the viscida thickness $h$ and a constitutive relation for the bending moment $\mathcal{M}$. This will be done in the following section by non-dimensionalizing the equations and exploiting the smallness of the slenderness parameter $\epsilon$.

\section{Perturbative analysis}

\subsection{Non-dimensionalization}

We suppose that curvatures are of magnitude $1 / L$, while the timescale is fixed by a balance between the surface tension and viscosity, thus indicating the scalings

$$
\begin{array}{rlrlrl}
s & =L(0) s^{\prime}, & n=\epsilon L(0) n^{\prime}, & t=\frac{\epsilon L(0) \mu}{\gamma} t^{\prime}, & \theta=\theta^{\prime}, \\
\boldsymbol{r}_{\boldsymbol{c}}=L(0) \boldsymbol{r}_{\boldsymbol{c}}{ }^{\prime}, & h=\epsilon L(0) h^{\prime}, & \boldsymbol{u}=\frac{\gamma}{\epsilon \mu} \boldsymbol{u}^{\prime}, & \boldsymbol{v}=\frac{\gamma}{\epsilon \mu} \boldsymbol{v}^{\prime}, \\
p=\frac{\gamma}{\epsilon L(0)} p^{\prime}, & \kappa=\frac{1}{L(0)} \kappa^{\prime}, & \kappa^{ \pm}=\frac{1}{L(0)} \kappa^{ \pm \prime}, & L=L(0) L^{\prime} .
\end{array}
$$

We non-dimensionalize the components of the stress tensor via

$$
\sigma_{s s}=\frac{\gamma}{\epsilon L(0)} \sigma_{s s}^{\prime}, \quad \sigma_{s n}=\frac{\gamma}{L(0)} \sigma_{s n}^{\prime}, \quad \sigma_{n n}=\frac{\gamma}{L(0)} \sigma_{n n}^{\prime},
$$

so that

$$
\begin{aligned}
\sigma_{s s}^{\prime} & =-p^{\prime}+\frac{2}{l^{\prime}}\left(\frac{\partial u_{s}^{\prime}}{\partial s^{\prime}}-u_{n}^{\prime} \kappa^{\prime}\right), \\
\epsilon^{2} \sigma_{s n}^{\prime} & =\frac{1}{l^{\prime}}\left(l^{\prime} \frac{\partial u_{s}^{\prime}}{\partial n^{\prime}}+\epsilon \kappa^{\prime} u_{s}^{\prime}+\epsilon \frac{\partial u_{n}^{\prime}}{\partial s^{\prime}}\right), \\
\epsilon^{2} \sigma_{n n}^{\prime} & =-\epsilon p^{\prime}+2 \frac{\partial u_{n}^{\prime}}{\partial n^{\prime}},
\end{aligned}
$$

where $l^{\prime}=1-\epsilon \kappa^{\prime} n^{\prime}$. The dimensionless net force components and bending moment are defined by

$$
\mathcal{T}=\epsilon^{2} \gamma \mathcal{T}^{\prime}, \quad \mathcal{F}=\epsilon^{2} \gamma \mathcal{F}^{\prime}, \quad \mathcal{A}=\epsilon^{2} \gamma \mathcal{A}^{\prime} \quad \mathcal{B}=\epsilon^{2} \gamma \mathcal{B}^{\prime} \quad \mathcal{M}=\epsilon^{2} \gamma L(0) \mathcal{M}^{\prime}
$$

\subsection{Distinguished limit}

Before we can pose asymptotic expansions for the dependent variables, we have to decide how to scale the applied pressure drop $\Delta P$, and it transpires that there are two 
distinguished limits. We assume that the dimensionless pressure drop

$$
\mathcal{P}=\frac{\Delta P L(0)}{\epsilon^{2} \gamma}
$$

is an order-one parameter, so that the shape of the viscida is determined by a balance between the applied pressure and surface tension. As a consequence of this assumption, we will find that the pressure drop has no effect on the thickness of the viscida, which therefore evolves purely under the action of surface tension.

The alternative limit occurs if $\mathcal{P}=O\left(1 / \epsilon^{2}\right)$, in which case the larger pressure drop causes the viscida instantaneously to form a circular arc, whose radius and thickness then evolve under both surface tension and pressure drop. This regime has been studied for two-dimensional configurations by van de Fliert et al. (1995), and is also relevant to circular hollow fibres (Fitt et al. 2001, 2002), where the applied pressure is used to control the rate at which the hole closes. However, it is evidently inappropriate when we wish to describe non-trivial evolution in the shape of the viscida.

In summary, we consider the asymptotic limit in which pressure affects the bending but not the stretching of the viscida. In the alternative regime, the pressure drop does influence the stretching and, therefore, dominates the bending. We note that Ribe (2001) combines both distinguished limits in a uniformly valid asymptotic model that describes both bending and stretching.

Having chosen the appropriate scaling for $\Delta P$, we now expand all dependent variables as regular asymptotic expansions of the form $\theta^{\prime}=\theta^{(0)}+\epsilon \theta^{(1)}+\epsilon^{2} \theta^{(2)}+\cdots$, and drop primes for clarity of notation. We omit all the details of the analysis which are analogous to those given in Buckmaster et al. (1975) and Buckmaster \& Nachman (1978).

\subsection{Leading-order equations}

The leading-order global force balances, (2.30) and (2.31) imply that

$$
\mathcal{A}^{(0)}=\mathcal{A}_{0}-\mathcal{P} y^{(0)}, \quad \mathcal{B}^{(0)}=\mathcal{B}_{0}+\mathcal{P} x^{(0)},
$$

where $\mathcal{A}_{0}$ and $\mathcal{B}_{0}$ are arbitrary functions of time, and $x^{(0)}$ and $y^{(0)}$ are the leading-order $x$ - and $y$-coordinates for the position of the centre-line of the sheet, that is,

$$
\frac{\partial x^{(0)}}{\partial s}=\cos \theta^{(0)}, \quad \frac{\partial y^{(0)}}{\partial s}=\sin \theta^{(0)} .
$$

The global torque balance, (2.32), thus gives

$$
\frac{\partial \mathcal{M}^{(0)}}{\partial s}=-\mathcal{A}_{0} \sin \theta^{(0)}+\mathcal{B}_{0} \cos \theta^{(0)}+\frac{\mathcal{P}}{2} \frac{\partial}{\partial s}\left(x^{(0)^{2}}+y^{(0)^{2}}\right) .
$$

This is the generalization of the governing equation obtained in Buckmaster \& Nachman (1978) to account for internal pressurization.

Perturbative analysis of the governing equations (2.10)-(2.12) and boundary conditions (2.16) and (2.17), and use of (2.9) reveals that the leading-order tangential velocity is independent of $n$ and thus the flow is extensional: $u_{s}^{(0)}=u_{s}^{(0)}(s, t)$. Then an equation representing conservation of mass is obtained in the form

$$
\frac{\partial h^{(0)}}{\partial t}+\frac{\partial}{\partial s}\left(\tilde{u}_{s}^{(0)} h^{(0)}\right)=0
$$

where $\tilde{u}_{s}^{(0)}=u_{s}^{(0)}-v_{s}^{(0)}$ is the tangential velocity of the fluid relative to the centre-line. 
We also obtain the equation

$$
h^{(0)} \frac{\partial \tilde{u}_{s}^{(0)}}{\partial s}=-\frac{1}{2}
$$

which represents a balance between surface tension and the viscous stress in the $s$ direction. We can therefore express (3.11) as

$$
\frac{\mathrm{D} h^{(0)}}{\mathrm{D} t}=\frac{1}{2}
$$

where

$$
\frac{\mathrm{D}}{\mathrm{D} t}=\frac{\partial}{\partial t}+\tilde{u}_{s}^{(0)} \frac{\partial}{\partial s}
$$

is the leading-order material derivative. Hence we find that the thickness of each material section through the viscida increases at a uniform rate under surface tension - as anticipated the pressure drop has no effect on this evolution.

Finally, we obtain an expression for the leading-order bending moment:

$$
\mathcal{M}^{(0)}=-\frac{h^{(0)^{3}}}{3} \frac{\mathrm{D}}{\mathrm{D} t}\left(\frac{\partial \theta^{(0)}}{\partial s}\right),
$$

as in Buckmaster \& Nachman (1978), and so the governing equation (3.10) for the evolution of the centre-line may be written as

$$
\frac{\partial}{\partial s}\left[\frac{h^{(0)^{3}}}{3} \frac{\mathrm{D}}{\mathrm{D} t}\left(\frac{\partial \theta^{(0)}}{\partial s}\right)\right]=\mathcal{A}_{0} \sin \theta^{(0)}-\mathcal{B}_{0} \cos \theta^{(0)}-\frac{\mathcal{P}}{2} \frac{\partial}{\partial s}\left(x^{(0)^{2}}+y^{(0)^{2}}\right) .
$$

Equations (3.13) and (3.16), given initial conditions, $\theta(s, 0), h(s, 0)$, and boundary conditions $(2.18)-(2.20)$, will determine $\theta(s, t)$ and $h(s, t)$.

\subsection{A uniformly thick viscida}

Dropping superscripts on leading-order variables, let us now consider a profile which is initially uniform in thickness. By defining $\epsilon$ specifically as the ratio between the initial thickness and length of the viscida, we may then choose $h(s, 0)=1$. The solution of (3.13) in this case is simply

$$
h=1+t / 2,
$$

and, by conservation of mass, the length of the viscida is given by

$$
L=\frac{1}{1+t / 2} \text {. }
$$

The ratio of tube thickness to circumference is therefore given by

$$
\frac{h}{L}=(1+t / 2)^{2}
$$

which implies that for sufficiently large time, the assumption of a thin viscida will fail. However, we are interested in profiles for which the final shape will have a thin wall, in which case the thin-walled assumption will be true throughout the evolution.

Since the sheet length varies with time, it is convenient to convert to a time-dependent coordinate system $(\xi, \tau)$, defined by

$$
\xi=(1+\tau / 2) s, \quad \tau=t .
$$

It is easily shown that $\xi$ is actually a Lagrangian coordinate that labels material sections 
of the viscida. We can therefore identify $\partial / \partial \tau$ with the material derivative $\mathrm{D} / \mathrm{D} t$ and hence write (3.16) in the form

$$
\frac{\partial}{\partial \tau}\left[(1+\tau / 2) \frac{\partial^{2} \theta}{\partial \xi^{2}}\right]=A(\tau) \sin \theta+B(\tau) \cos \theta-\frac{3 \mathcal{P}}{2(1+\tau / 2)^{5}} \frac{\partial}{\partial \xi}\left(X^{2}+Y^{2}\right) .
$$

Here the arbitrary functions $A$ and $B$ are defined by

$$
A=\frac{3 \mathcal{A}_{0}}{(1+\tau / 2)^{4}}, \quad B=-\frac{3 \mathcal{B}_{0}}{(1+\tau / 2)^{4}},
$$

and $X=x / L, Y=y / L$ are scaled Cartesian coordinates with respect to which the length of the sheet remains constant, specifically,

$$
\frac{\partial X}{\partial \xi}=\cos \theta, \quad \frac{\partial Y}{\partial \xi}=\sin \theta .
$$

Equations (3.21) and (3.23) govern the leading-order evolution of the viscida centreline. They require one initial condition for $\theta(\xi, 0)$ and the dimensionless forms of the boundary conditions (2.18)-(2.20), namely

$$
\theta(0, \tau)=0, \quad \theta(1, \tau)=2 \pi, \quad \int_{0}^{1} \cos \theta \mathrm{d} \xi=\int_{0}^{1} \sin \theta \mathrm{d} \xi=0 .
$$

Finally, we have to eliminate the arbitrary rigid-body translation by fixing the origin, for example by setting

$$
X(0, \tau)=X^{*}(\tau), \quad Y(0, \tau)=Y^{*}(\tau),
$$

where $X^{*}$ and $Y^{*}$ are specified. The six boundary conditions (3.24) and (3.25) in principle allow us to solve the fourth-order (in space) equation (3.21), determining the arbitrary functions $A$ and $B$ as part of the solution. We will show in the next section how $A$ and $B$ may be obtained instantaneously.

\subsection{Solution procedure}

Integration of (3.23) gives

$$
X=X_{0}+X^{*}, \quad Y=Y_{0}+Y^{*},
$$

where

$$
X_{0}=\int_{0}^{\xi} \cos \theta(\hat{\xi}, \tau) \mathrm{d} \hat{\xi}, \quad Y_{0}=\int_{0}^{\xi} \sin \theta(\hat{\xi}, \tau) \mathrm{d} \hat{\xi} .
$$

Without loss of generality, we choose the origin of our coordinate system such that

$$
X^{*}=-\overline{X_{0}}, \quad Y^{*}=-\overline{Y_{0}},
$$

where we define the cross-sectional average of any function $\varphi(\xi, \tau)$ by

$$
\bar{\varphi}=\int_{0}^{1} \varphi \mathrm{d} \xi .
$$


The surface-tension-driven evolution of a two-dimensional annular viscous tube 11 It follows that the origin lies at the centre-of-mass, that is, $\bar{X}=\bar{Y}=0$, and hence that $\overline{\mathcal{A}}$ and $\overline{\mathcal{B}}$ are given by

$$
\begin{aligned}
& \overline{\mathcal{A}}=\int_{0}^{1} \mathcal{A}(\xi, \tau) \mathrm{d} \xi=\mathcal{A}_{0}=A(1+\tau / 2)^{4} / 3 \\
& \overline{\mathcal{B}}=\int_{0}^{1} \mathcal{B}(\xi, \tau) \mathrm{d} \xi=\mathcal{B}_{0}=-B(1+\tau / 2)^{4} / 3 .
\end{aligned}
$$

Thus $\mathcal{A}_{0}$ and $\mathcal{B}_{0}$ are the total tensions in the sheet in the $X$ - and $Y$-directions respectively.

By integrating (3.21) with respect to $\xi$ and using conditions $(3.24 a, b)$, we obtain

$$
(1+\tau / 2) \theta_{\xi \tau}+\frac{1}{2} \theta_{\xi}=A Y+B X-\frac{3 \mathcal{P}}{2(1+\tau / 2)^{5}}\left(X^{2}+Y^{2}-\overline{X^{2}}-\overline{Y^{2}}\right)+\pi
$$

Multiplying this equation by $(X, Y)$ and integrating over the length of the sheet yields two equations to determine $A$ and $B$, namely

$$
\begin{aligned}
\left(\begin{array}{ll}
\overline{X Y} & \overline{X^{2}} \\
\overline{Y^{2}} & \overline{X Y}
\end{array}\right)\left(\begin{array}{l}
A \\
B
\end{array}\right)=\frac{1}{2} \int_{0}^{1}(2 \pi \xi-\theta)\left(\begin{array}{c}
\cos \theta \\
\sin \theta
\end{array}\right) \mathrm{d} \xi & \\
& +\frac{3 \mathcal{P}}{2(1+\tau / 2)^{5}}\left(\frac{\overline{X^{3}}+\overline{X Y^{2}}}{X^{2} Y}+\overline{Y^{3}}\right)
\end{aligned}
$$

Equation (3.32) may be integrated with respect to $\xi$ once more to give

$$
\begin{aligned}
\theta_{\tau}=\frac{2 \pi \xi-\theta}{2(1+\tau / 2)} & +\frac{A}{1+\tau / 2} \int_{0}^{\xi} Y(\hat{\xi}, \tau) \mathrm{d} \hat{\xi}+\frac{B}{1+\tau / 2} \int_{0}^{\xi} X(\hat{\xi}, \tau) \mathrm{d} \hat{\xi} \\
& -\frac{3 \mathcal{P}}{2(1+\tau / 2)^{6}}\left(\int_{0}^{\xi} X(\hat{\xi}, \tau)^{2}+Y(\hat{\xi}, \tau)^{2} \mathrm{~d} \hat{\xi}-\left(\overline{X^{2}}+\overline{Y^{2}}\right) \xi\right)
\end{aligned}
$$

Hence, given $\theta(\xi, 0)$ and using (3.33) to determine $A$ and $B$ at each instant, we can solve (3.34) for $\theta(\xi, \tau)$, for example by a time-stepping scheme.

For profiles with any rotational symmetry (i.e. profiles for which $\theta(1 / n+\xi)=\theta(\xi)+$ $2 \pi / n$ for some integer $n$ ), and hence also shapes with more than one line of reflectional symmetry, it is a straightforward but tedious exercise to show that

$$
\begin{array}{r}
\int_{0}^{1}(2 \pi \xi-\theta)\left(\begin{array}{c}
\cos \theta \\
\sin \theta
\end{array}\right) \mathrm{d} \xi=\mathbf{0}, \\
\bar{X}=\overline{X^{3}}=\overline{X Y^{2}}=\overline{X^{2} Y}=\overline{Y^{3}}=0,
\end{array}
$$

and hence that $A=B=0$. This is physically sensible since we expect the resultant force to be zero for shapes with rotational symmetry. In fact, it is more surprising that $A$ and $B$ might not be zero for asymmetric shapes. We give one example of such a situation below, but otherwise we restrict our attention to the industrially relevant case of a rotationally symmetric profile, for which the governing equation (3.21) simplifies to

$$
\left[(1+\tau / 2) \theta_{\xi \xi}\right]_{\tau}=-\frac{3 \mathcal{P}}{2(1+\tau / 2)^{5}}\left(X^{2}+Y^{2}\right)_{\xi}
$$




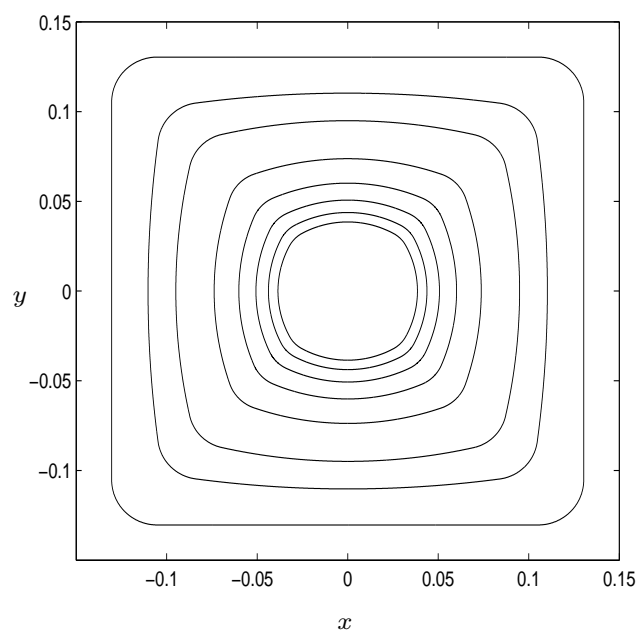

(a)

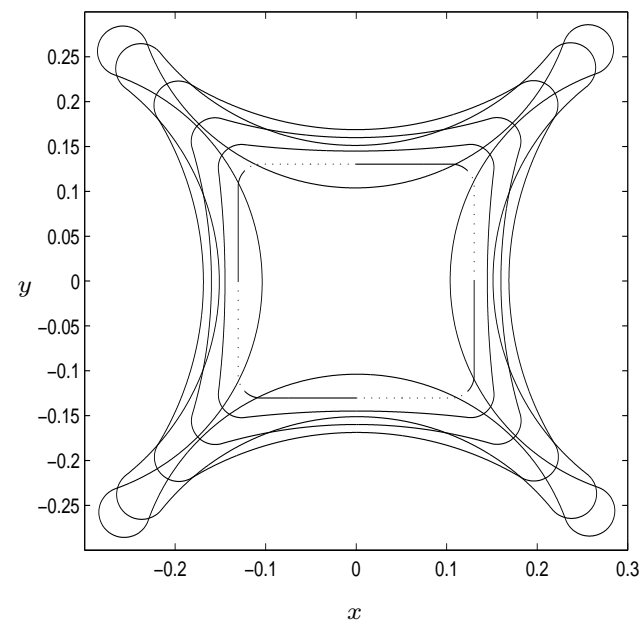

(b)

FiguRE 3. Evolution (in Eulerian coordinates) of an initially square centre-line with rounded corners under surface tension with no applied pressure. (a) Positive time evolution with $\tau=0,0.5,1,2,3,4,5,6$. (b) Negative time evolution with $\tau=0,-0.3,-0.6,-0.9,-1.15,-1.3$.

\section{Time-dependent solutions}

\subsection{Zero applied pressure}

When there is no applied pressure, (3.37) is easily solved analytically in the form

$$
\theta(\xi, \tau)=\frac{\theta(\xi, 0)-2 \pi \xi}{(1+\tau / 2)}+2 \pi \xi
$$

We immediately note that $\theta \rightarrow 2 \pi \xi$ as $\tau \rightarrow \infty$, implying that the viscida will tend towards a circular shape regardless of its initial configuration. We also observe that, since $\theta$ depends only algebraically on $\tau$, the problem is surprisingly well posed for negative time. We can thus use (4.1) to solve explicitly the inverse problem of determining the initial shape that will evolve to a required final shape.

In figure 3 we show the evolution of an initial square profile with rounded corners (so the curvature is bounded everywhere). As time increases, the angles between the edges increase and the profile evidently approaches a circle. For negative time, the angles decrease, causing the corners to stretch out and form 'fingers', which pinch off when $\tau \approx-1.3$. This behaviour may be explained by differentiating (4.1) with respect to $\tau$ to obtain

$$
\theta_{\tau}=\frac{2 \pi \xi-\theta(\xi, \tau)}{2(1+\tau / 2)}
$$

Thus portions of the viscida which are further from a circular profile will evolve more quickly and, specifically, the angle $\theta$ increases with time where $\theta<2 \pi \xi$ and decreases where $\theta>2 \pi \xi$. For our initial square set-up, this implies that for decreasing time the angle will decrease in the dotted parts of the initial profile in figure 3(b) and increase in the solid portions, hence causing the corners to stretch out. We re-emphasize that the shapes illustrated in figure 3(b) will evolve to the desired square shape under surface tension and hence are explicit solutions of the inverse problem posed in the Introduction.

We note that our theory is valid provided the thickness of the viscida remains every- 


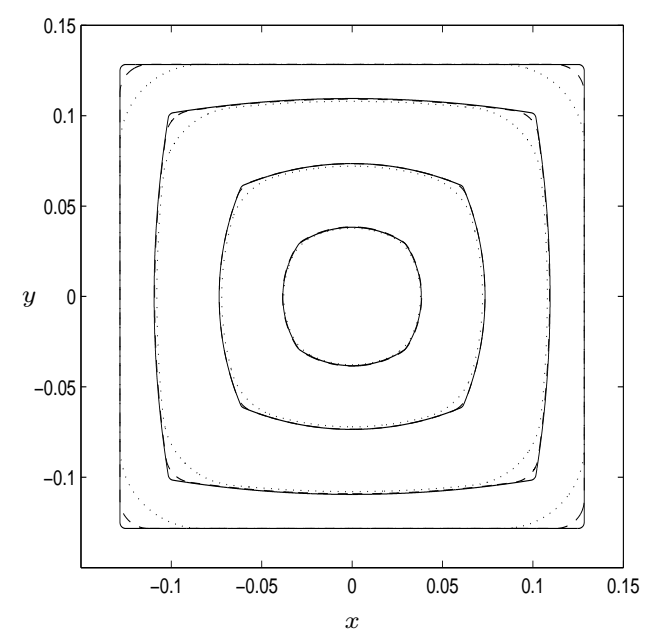

FIGURE 4. Evolution (in Eulerian coordinates) under surface tension with no applied pressure of an initially square profile with rounded corners. The initial radius of curvature is $0.02 L(0)$ (solid), $0.1 L(0)$ (dashed), $0.3 L(0)$ (dotted) and the profile is shown at dimensional times $0,0.5 \epsilon L(0) \mu / \gamma, 2 \epsilon L(0) \mu / \gamma, 6 \epsilon L(0) \mu / \gamma$.

where much smaller than its radius of curvature. If this condition is violated our theory is no longer valid and analysis of the full two-dimensional Stokes equations is required. Since we are concerned with the production of polygonal profiles, it is therefore important that the corners of such shapes are sufficiently smoothed off to ensure that the profile satisfies this assumption. However, in figure 4 we show the effect of increasing the curvature to approach an initially square configuration. We conclude that increasing the curvature does not generate any adverse effects in the global profile evolution and therefore infer that our analysis may be used to describe the global evolution of a viscida which contains small regions of high curvature. The local behaviour in the small regions will be described by an inner problem, for which the full Stokes equations must be considered, but such inner behaviour will not influence the global sheet evolution.

Figures 5 and 6 highlight the same features for initial triangular and pentagonal profiles, showing evolution towards a circular profile for forward time and the stretching out of the corners for inverse time. We observe that generically the negative-time solutions fail via a change in topology before the critical time $\tau=-2$ at which the viscida length tends to infinity. We notice that the greater the number of vertices the profile has, the greater the internal angle of each vertex and hence the larger the inverse time it takes to pinch off. In the limit as the number of vertices tends to infinity, the initial profile approaches a circle, which is preserved under positive and negative time evolution and hence never pinches off.

Finally, we illustrate the danger of assuming $A=B=0$ for an asymmetric profile. In figure 7 we show the evolution of a profile which has no symmetry, if we still choose $A=B=0$, that is, the evolution as predicted by (4.1). It is evident that the boundary condition enforcing the joining of the ends of the viscida is violated in this case, especially for negative times. The correct evolution, obtained by solving (3.33) and (3.34) numerically, is shown in figure 8, with the relation of both $A$ and $B$ to time required to satisfy all four boundary conditions (3.24) in this case, displayed in figure 9 . We note 


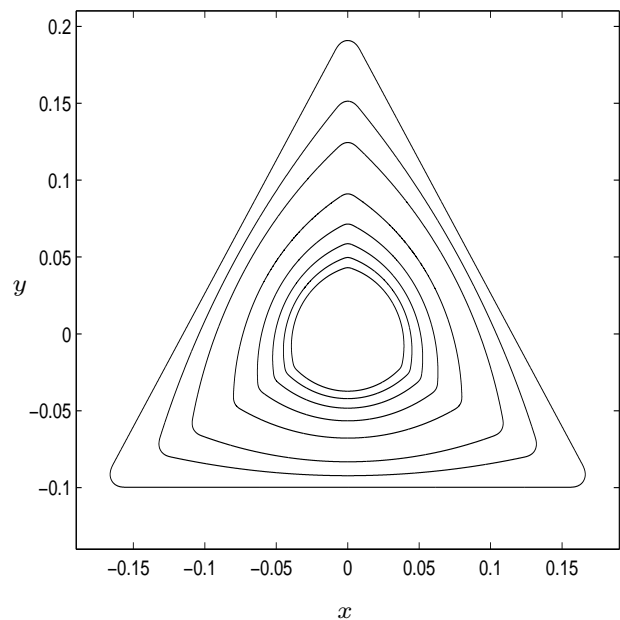

(a)

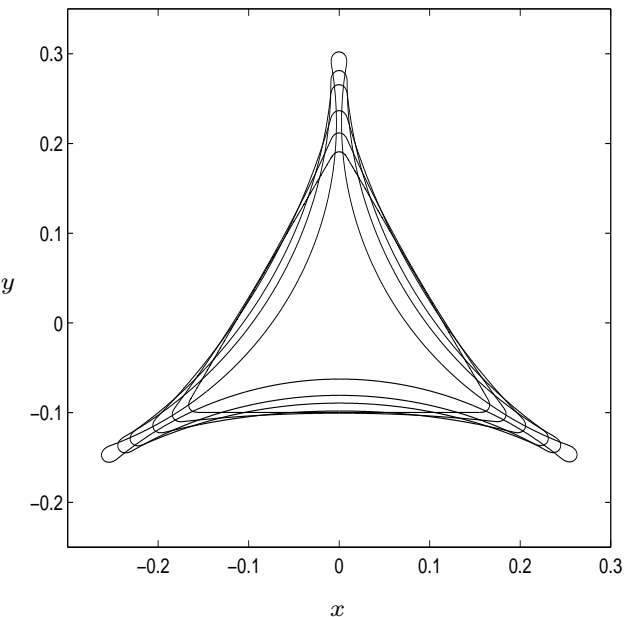

(b)

FiguRE 5. Evolution (in Eulerian coordinates) of an initially triangular centre-line with rounded corners under surface tension with no applied pressure. (a) Positive time evolution with $\tau=0,0.5,1,2,3,4,5,6$. (b) Negative time evolution with $\tau=0,-0.2,-0.4,-0.6,-0.7,-0.83$.

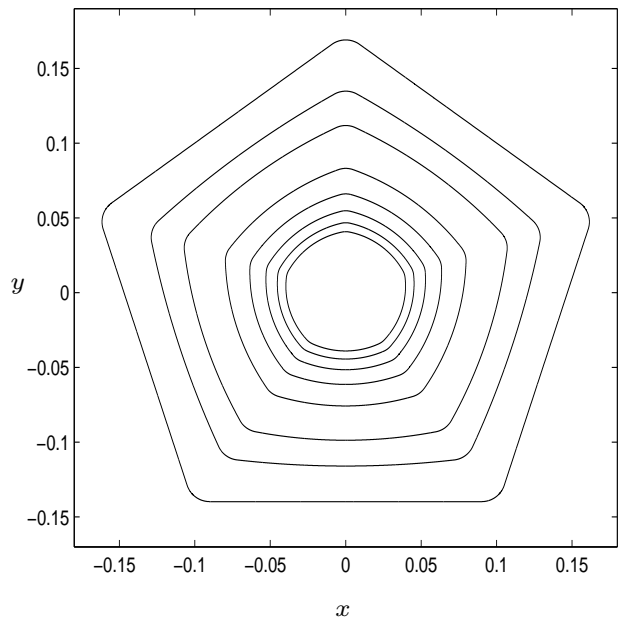

(a)

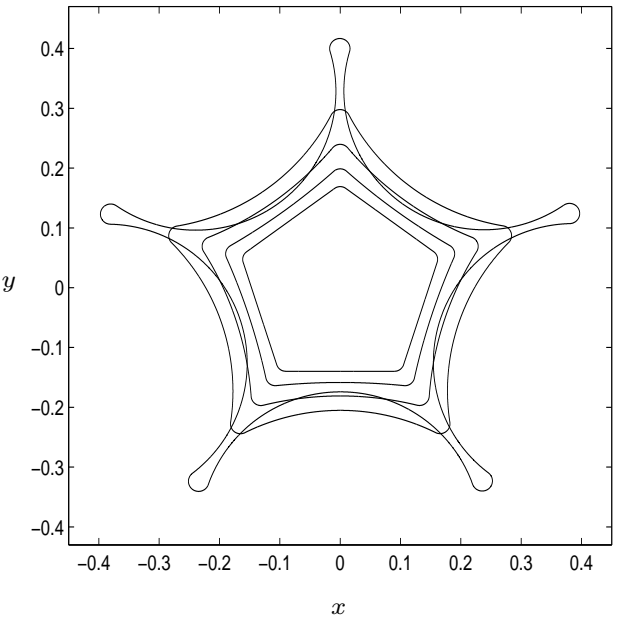

(b)

FIgURE 6. Evolution (in Eulerian coordinates) of an initially pentagonal centre-line with rounded corners under surface tension with no applied pressure. (a) Positive time evolution with $\tau=0,0.5,1,2,3,4,5,6$. (b) Negative time evolution with $\tau=0,-0.3,-0.6,-0.9,-1.38$.

that both $A$ and $B$ tend towards zero when $\tau \rightarrow \infty$, as the profile approaches a circle, but increase dramatically for negative values of $\tau$.

\subsection{Non-zero applied pressure}

For the case where $\mathcal{P}$ is non-zero, explicit solution is no longer possible but we may instead perform a forward time-stepping scheme on (3.34), or (3.37) if the profile is rotationally symmetric. 


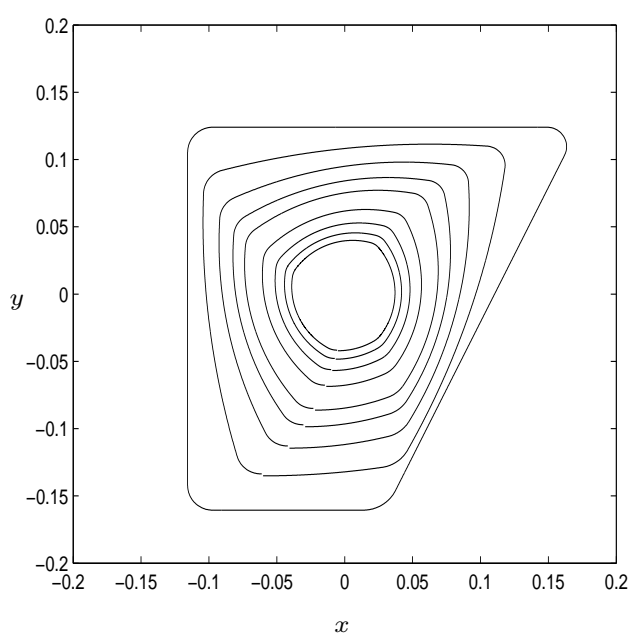

(a)

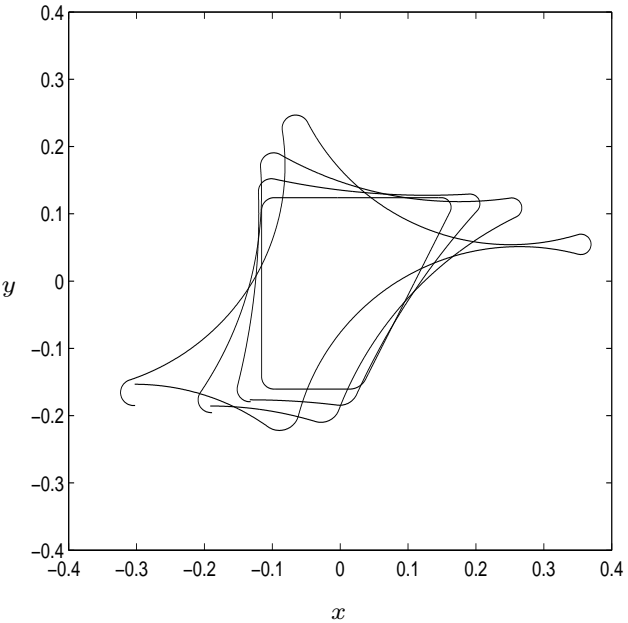

(b)

FIgURE 7. Predicted evolution (in Eulerian coordinates) of an asymmetric profile under surface tension with no applied pressure assuming $A=B=0$. (a) Positive time evolution with $\tau=0,0.5,1,1.5,2,3,4,5,6$. (b) Negative time evolution with $\tau=0,-0.3,-0.6,-0.95$.

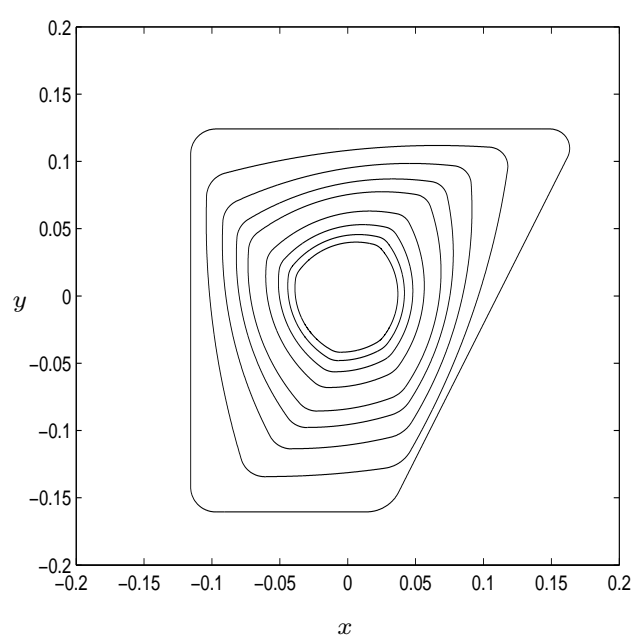

(a)

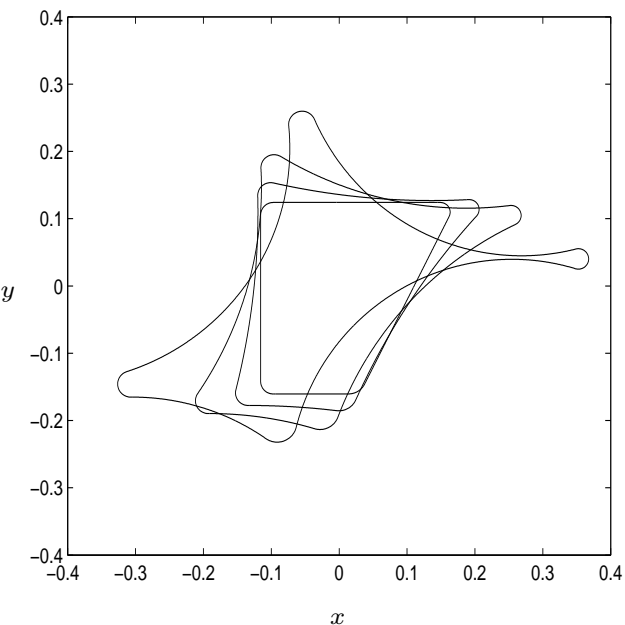

(b)

FIGURE 8. Correct evolution (in Eulerian coordinates) of an asymmetric profile under surface tension with no applied pressure. (a) Positive time evolution with $\tau=0,0.5,1,1.5,2,3,4,5,6$. (b) Negative time evolution with $\tau=0,-0.3,-0.6,-0.95$.

In figure 10 we show the positive-time evolution of an initially square viscida (with rounded corners) under an applied pressure (a) $\mathcal{P}=-1500$, (b) $\mathcal{P}=+1500$. Despite these apparently large dimensionless values, in neither case does the evolution differ dramatically from the zero-pressure behaviour shown in figure 3(a). As time increases, the pressure acts over a shrinking circumference, while the viscida becomes thicker and hence harder to deform. These two effects conspire to reduce the effect of pressure at positive values of $\tau$. They are reflected in the factor $1 /(1+\tau / 2)^{5}$ that multiplies the 


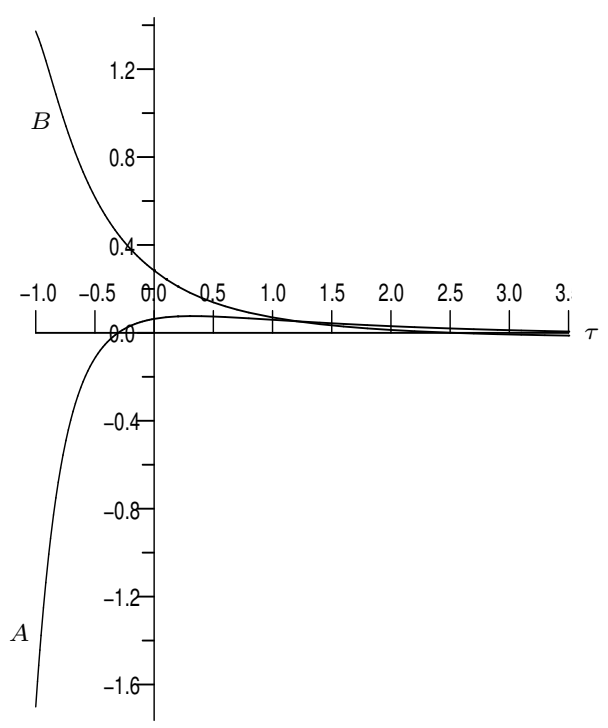

Figure 9. Relation of $A$ and $B$ to time $\tau$ for the evolution of the initial asymmetric profile in figure 8 .

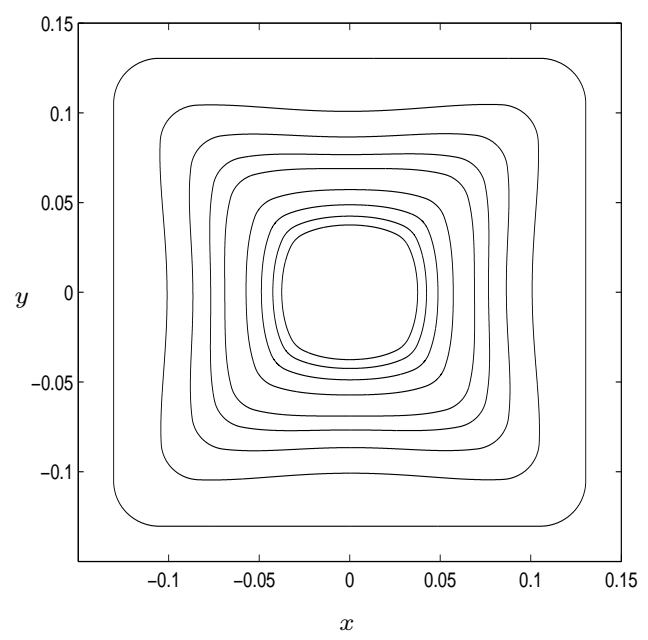

(a)

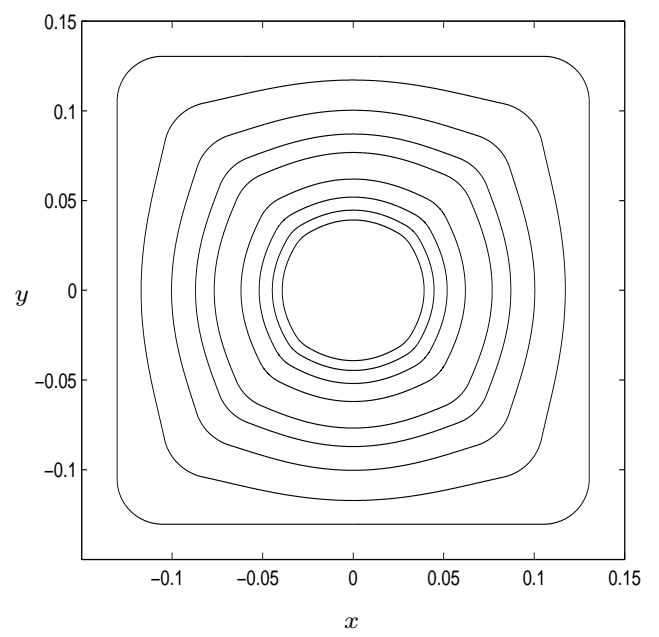

(b)

Figure 10. Evolution (in Eulerian coordinates) of an initially square centre-line with rounded corners under surface tension and applied pressure. (a) Negative pressure $\mathcal{P}=-1500$ at $\tau=0,0.5,1.0,1.5,2.0,3.0,4.0,5.0,6.0$. (b) Positive pressure $\mathcal{P}=1500$ at $\tau=0,0.5,1.0,1.5,2.0,3.0,4.0,5.0,6.0$.

pressure term in (3.37) and quickly renders the applied pressure negligible for positive time.

Figure 10(b) shows that a positive applied pressure serves to accelerate the evolution towards a circle. However, when a negative pressure is applied, figure 10(a) shows that the applied suction causes the straight edges initially to buckle inwards towards the centre. As noted above, the applied pressure soon becomes negligible as $\tau$ increases, so surface tension causes the centre-line to approach a circle, the first symptom of which is 


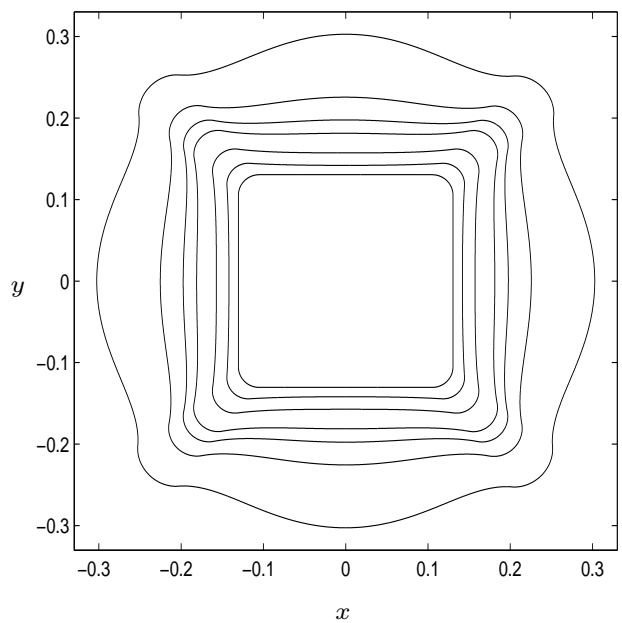

(a)

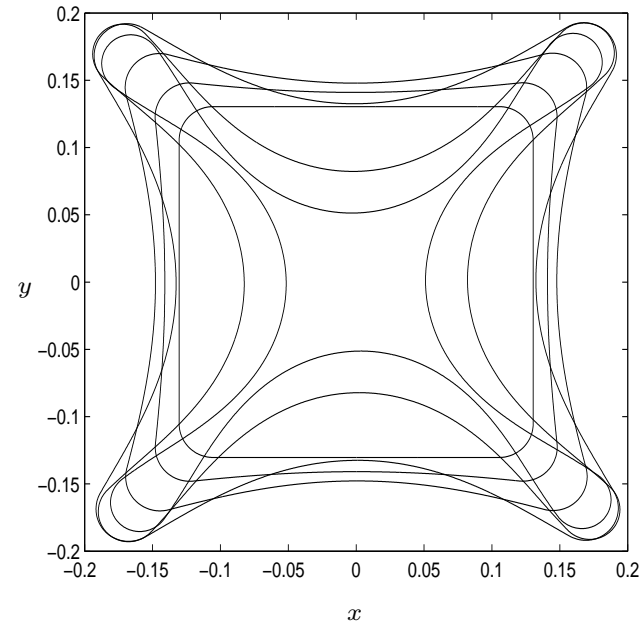

(b)

FiguRE 11. Negative-time evolution (in Eulerian coordinates) of an initially square centre-line with rounded corners under surface tension and applied pressure. (a) Negative pressure $\mathcal{P}=-200$ at $\tau=0,-0.2,-0.4,-0.6,-0.7,-0.8,-1.0$. (b) Positive pressure $\mathcal{P}=100$ at $\tau=0,-0.25,-0.5,-0.75,-0.9,-0.95$.

the corners being pulled inwards. The competition between suction and surface tension means that, after a certain time, the profile appears to return to a shape that closely resembles a scaled version of the original profile. We will return to this idea in $\S 6.2$.

For negative values of $\tau$, the $1 /(1+\tau / 2)^{5}$ factor grows rapidly so that the applied pressure dominates the behaviour as $\tau$ approaches -2 . Hence the magnitude of $\mathcal{P}$ need not be as large as for the forward-time solutions to have a marked effect on the profile evolution.

Figure 11 illustrates the negative-time evolution of a square with rounded corners for inverse time due to (a) negative pressure $\mathcal{P}=-200$, (b) positive pressure $\mathcal{P}=100$. In case (a), we see that the applied suction causes the edges to bulge outwards and the profile to approach a circle. This is unsurprising when we recall that an applied suction causes the edges to buckle inwards initially for positive values of $\tau$. The practical implication is that by making $\mathcal{P}$ sufficiently large and negative, we can suppress the formation of fingers and hence prevent the tube from pinching off in negative time. The application of a negative pressure may thus enable the production of shapes which are impossible to obtain using surface tension alone.

When $\mathcal{P}$ is positive, the negative-time formation of fingers is exacerbated and the time to pinch-off is thus decreased. To produce a square at a later time therefore requires a more highly deformed initial shape if we apply a positive pressure.

\section{Steady solutions}

Re-examining figures 10 (a) and 11(a), we observe that a negative pressure slows down the evolution of the centre-line, so that the initial square shape is roughly preserved, at least for relatively small changes in $\tau$. This leads us to pose the question: 'Are there shapes that are preserved exactly under the application of a suitable applied pressure?' Such shapes correspond to steady solutions of the Lagrangian equation (3.37), although 


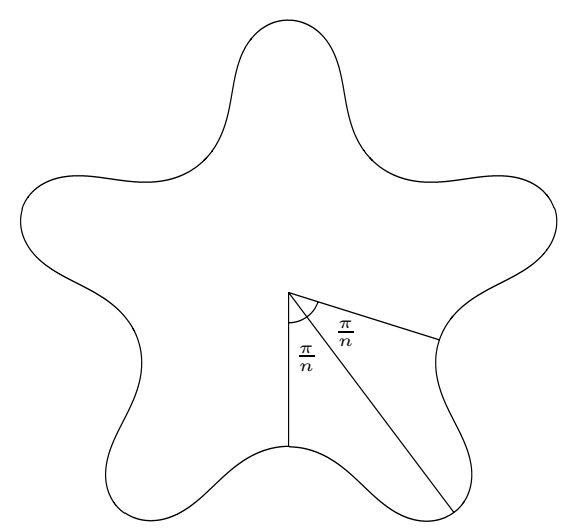

Figure 12. A viscida centre-line with $n=5$ degrees of rotational symmetry, each of the five regions possessing reflection symmetry.

the Eulerian circumference of the viscida scales with $\tau$ according to (3.18). Such solutions can only exist if the applied pressure is a suitable function of $\tau$, namely

$$
\mathcal{P}=-\frac{p(1+\tau / 2)^{5}}{3},
$$

where $p$ is a constant. At first glance, it may not seem physically practicable to vary $\mathcal{P}$ in this prescribed way, but we will show in $\S 6.2$ how the analysis given below can be generalized to applied pressures that do not satisfy (5.1).

We will continue to assume that the profile has $n$ degrees of rotational symmetry, so that $\theta(1 / n+\xi)=\theta(\xi)+2 \pi / n$. In addition, we suppose for simplicity that each of these $n$ regions has reflection symmetry, so that $\theta(1 / 2 n+\xi)=2 \pi / n-\theta(1 / 2 n-\xi)$. A typical such profile is illustrated schematically in figure 12. As a result of these assumptions, we need only solve in the region $0 \leqslant \xi \leqslant 1 / 2 n$, and the remainder of the viscida may then be inferred by symmetry.

With $\theta$ independent of $\tau$, we integrate (3.37) to obtain

$$
\frac{\mathrm{d} \theta}{\mathrm{d} \xi}=p\left(X^{2}+Y^{2}\right)+2 C,
$$

where $C$ is a constant of integration. Interestingly, this equation also describes the deformation of a thin elastic tube under an applied pressure $p$ (see, for example Love 1927, article 275). It may be rearranged to

$$
\frac{\mathrm{d}}{\mathrm{d} \xi}(X \sin \theta-Y \cos \theta)=\frac{\mathrm{d}}{\mathrm{d} \xi}\left[\frac{p}{4}\left(X^{2}+Y^{2}\right)^{2}+C\left(X^{2}+Y^{2}\right)\right],
$$

and hence integrated once more with respect to $\xi$. We write the resulting differential equation in terms of a single dependent variable $R(\xi)=\sqrt{X^{2}+Y^{2}}$, representing the distance of the centre-line from the origin, as

$$
R^{\prime}(\xi)=\sqrt{1-\frac{\left(p R^{4} / 4+C R^{2}+D\right)^{2}}{R^{2}}},
$$

where $D$ is another integration constant. 


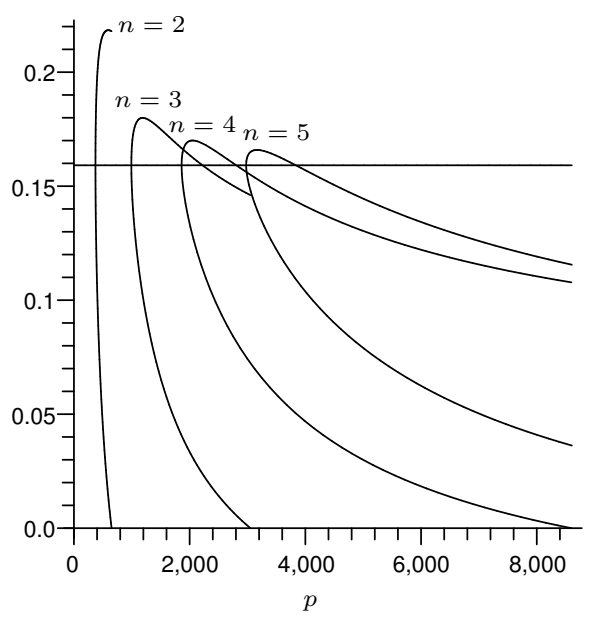

FIGURE 13. Bifurcation diagram showing the maximum and minimum radii, $R_{ \pm}$, versus pressure parameter $p$.

We can relate the two constants $C$ and $D$ to the maximum and minimum radii $R_{+}$ and $R_{-}$respectively, using the fact that $R^{\prime}=0$ when $R=R_{ \pm}$:

$$
\begin{aligned}
C & =\frac{1}{R_{+}+R_{-}}-\frac{p}{4}\left(R_{+}^{2}+R_{-}^{2}\right), \\
D & =\frac{R_{+} R_{-}}{R_{+}+R_{-}}+\frac{p}{4} R_{+}^{2} R_{-}^{2} .
\end{aligned}
$$

Then $R_{+}$and $R_{-}$can be evaluated in principle using the two conditions

$$
\begin{gathered}
p \int_{R_{-}}^{R_{+}} \frac{R^{2}}{R^{\prime}} \mathrm{d} R=\frac{\pi-C}{n}, \\
\int_{R_{-}}^{R_{+}} \frac{\mathrm{d} R}{R^{\prime}}=\frac{1}{2 n}
\end{gathered}
$$

where $R^{\prime}$ is given by (5.4); (5.7) represents the condition $\theta(1 / 2 n)-\theta(0)=\pi / n$ and (5.8) expresses conservation of arc-length.

The task of solving for $R_{ \pm}$in terms of $p$ is simplified by introducing the variables

$$
\bar{R}=\frac{R_{+}+R_{-}}{2}, \quad \nu=\frac{R_{+}-R_{-}}{R_{+}+R_{-}}, \quad \beta=p\left(\frac{R_{+}+R_{-}}{2}\right)^{3}, \quad r=\frac{2 R}{R_{+}+R_{-}},
$$

so that (5.7) and (5.8) decouple. First, (5.7) becomes an equation to determine $\nu$ in terms of $\beta$ :

where

$$
\int_{1-\nu}^{1+\nu}\left(\beta r^{2}+1-\beta\left(1+\nu^{2}\right)\right) G(r ; \nu, \beta) \mathrm{d} r=\frac{\pi}{n}
$$

$$
\begin{aligned}
G(r ; \nu, \beta)=\frac{1}{R^{\prime}} & =4 r[(1+\nu+r)(1-\nu+r)(1+\nu-r)(-1+\nu+r) \\
& \left.\left(2+\beta\left(1-\nu^{2}\right)+2 \beta r+\beta r^{2}\right)\left(2+\beta\left(1-\nu^{2}\right)-2 \beta r+\beta r^{2}\right)\right]^{-1 / 2} .
\end{aligned}
$$


Then (5.8) gives an equation for $\bar{R}$ in terms of $\beta$ :

$$
\bar{R}=\left[2 n \int_{1-\nu}^{1+\nu} G(r ; \nu(\beta), \beta)\right]^{-1}
$$

The original variables are then given parametrically by

$$
R_{ \pm}=\bar{R}(1 \pm \nu), \quad p=\frac{\beta}{\bar{R}^{3}} .
$$

Figure 13 shows a plot of $R_{ \pm}$versus $p$, illustrating how $n$-lobed solutions bifurcate from the circular profile $R=1 / 2 \pi$ as $p$ increases. Each branch ceases to exist when $R_{-}$becomes zero, although, as we shall see below, they typically pinch off before this occurs.

Once $R_{ \pm}$are determined, the centre-line profile $r(\xi)$ is given implicitly by integration of (5.11), that is,

$$
\xi=\bar{R} \int_{1-\nu}^{r} G(\hat{r} ; \nu, \beta) \mathrm{d} \hat{r}
$$

The profile $(X, Y)$ may then be determined parametrically as

$$
X=\bar{R} r \cos \phi(r), \quad Y=\bar{R} r \sin \phi(r),
$$

where $\phi$ is the plane polar coordinate angle obtained using (5.3),

$$
\phi(r)=\theta(r)-\arcsin \left[\frac{\beta(r-(1-\nu))+\left(2-2 \beta\left(1+\nu^{2}\right)\right)\left(r^{2}-(1-\nu)^{2}\right)}{4 r}\right]
$$

and, from (5.2),

$$
\theta(r)=\int_{1-\nu}^{r}\left(\beta \hat{r}^{2}+1-\beta\left(1+\nu^{2}\right)\right) G(\hat{r} ; \nu, \beta) \mathrm{d} \hat{r} .
$$

The integrals (5.7)-(5.14) may all be expressed in terms of incomplete elliptic integrals $\dagger$ but we found it more convenient to evaluate them numerically.

Figure 14 shows plots of steady $n$-lobed solutions for $n=2,3,4,5$ and various values of $p$. The generic behaviour is exemplified by the $n=3$ case. As $p$ increases, the viscida buckles so the larger radius $R_{+}$initially increases. As $p$ increases further and the viscida crumples, the outer radius starts to decrease, eventually becoming smaller than its initial value, as shown in figure 13. Each solution loses physical validity by pinching off before the inner radius reaches zero.

It is noticeable (see in particular $n=3$ and $n=4$ ) that some of the steady solutions shown in figure 14 closely resemble the rounded polygonal profiles that we are trying to produce. Furthermore, each of these corresponds to a value of $p$ close to the bifurcation. This suggests that we may be able to describe approximately polygonal shapes quite well using a weakly nonlinear theory, seeking solutions whose profiles are close to circular. We explore this possibility in the following section. 


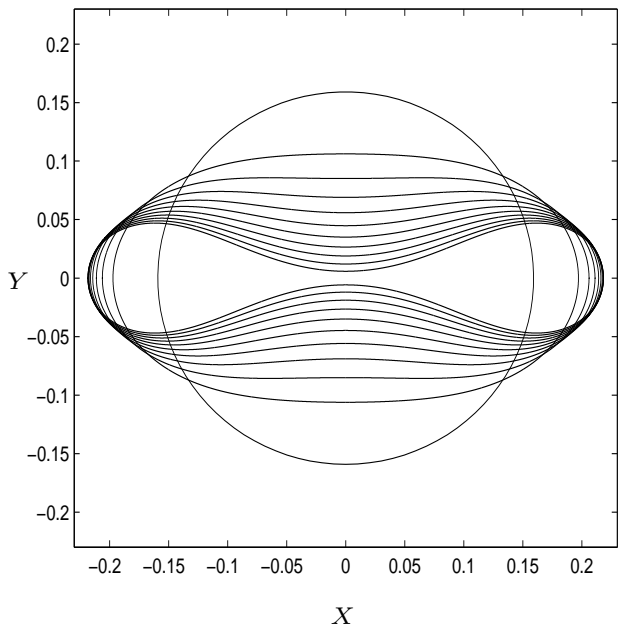

(a)

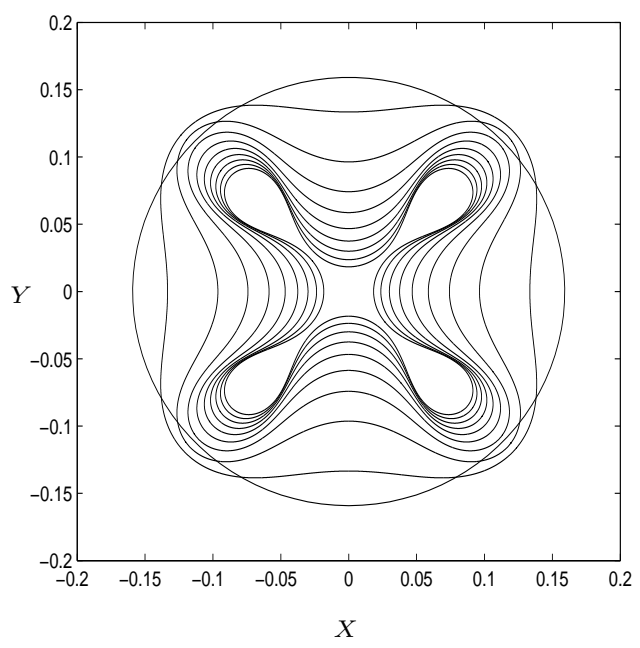

(c)

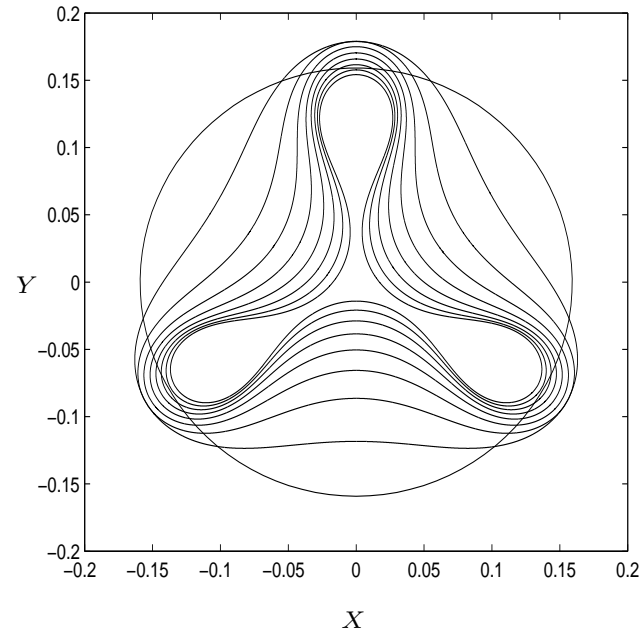

(b)

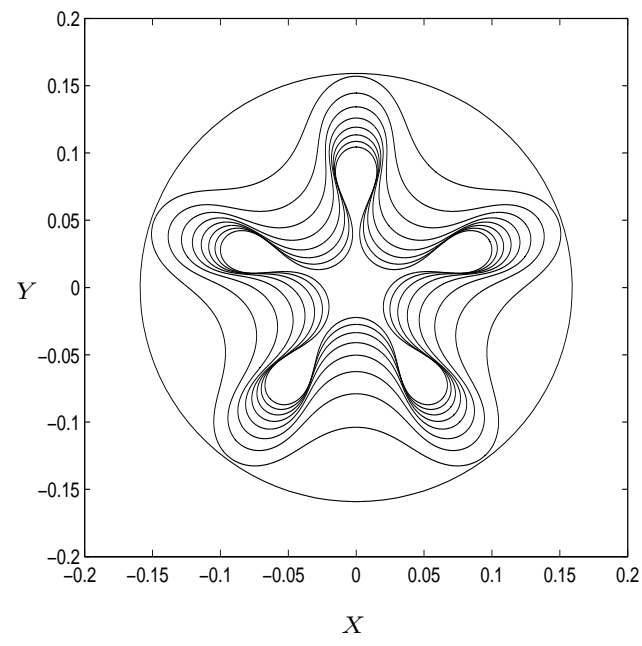

(d)

FIgURE 14. Nonlinear steady $n$-lobed solutions with $n=2,3,4,5$ with (a) $p=0,400,425,450, \ldots, 625, \quad$ (b) $p=0,1100,1300,1500, \ldots, 2500, \quad$ (c) $p=0,2000,2500,3000, \ldots, 6000$, (d) $p=0,4000,5000,6000, \ldots, 1100$.

\section{Weakly nonlinear analysis}

\subsection{Steady solutions}

We now tackle the steady solution equation (5.2) via a weakly nonlinear approach, seeking profiles that are small perturbations to a circle. Using the result

$$
\theta^{\prime}(\xi)=\frac{1-R^{\prime 2}-R R^{\prime \prime}}{R \sqrt{1-R^{\prime 2}}}
$$


we can write $(5.2)$ as

$$
\frac{1-R^{\prime 2}-R R^{\prime \prime}}{R \sqrt{1-R^{\prime 2}}}=p R^{2}+2 C .
$$

For an $n$-lobed solution, we apply the boundary conditions

$$
\begin{aligned}
R(0) & =R_{-}, \\
R(1 / 2 n) & =R_{+}, \\
R^{\prime}(0)=R^{\prime}(1 / 2 n) & =0, \\
\int_{0}^{1 / 2 n} \frac{1-R^{\prime 2}-R R^{\prime \prime}}{R \sqrt{1-R^{\prime 2}}} \mathrm{~d} \xi & =\frac{\pi}{2 n} .
\end{aligned}
$$

By linearizing about the circular solution $R^{(0)}(\xi)=1 / 2 \pi$, we easily discover that an $n$-lobed solution is born at the bifurcation value $p=p^{(0)}=4 \pi^{3}\left(n^{2}-1\right)$, in agreement with figure 13. Let us then suppose that $p$ is close to one of these critical values by setting

$$
p=4 \pi^{3}\left(n^{2}-1\right)+\delta^{2},
$$

where $|\delta| \ll 1$. We pose asymptotic expansions for $R$ and $C$ in the form

$$
\begin{aligned}
& R \sim 1 / 2 \pi+\delta a_{1} \cos (2 n \pi \xi)+\delta^{2} R^{(2)}(\xi)+\cdots, \\
& C \sim\left(3-n^{2}\right) \pi / 2+\delta^{2} C^{(2)}+\cdots,
\end{aligned}
$$

where the leading- and first-order solutions are easily determined from (6.2)-(6.6).

Our aim is to determine the first-order amplitude $a_{1}$, and it transpires that to do so one must continue the expansions to order $\delta^{3}$. We omit the details of the analysis, which yields the solvability condition

$$
a_{1}\left(a_{1}^{2}-\frac{n^{2}}{6 \pi^{5}\left(n^{2}-1\right)^{3}}\right)=0 .
$$

Hence we obtain a supercritical pitchfork bifurcation, as suggested by figure 13, with the maximum and minimum radii given by

$$
R_{ \pm} \sim \frac{1}{2 \pi} \pm \frac{n \sqrt{p-4 \pi^{3}\left(n^{2}-1\right)}}{\sqrt{6} \pi^{5 / 2}\left(n^{2}-1\right)^{3 / 2}}
$$

for an $n$-lobed profile when $p$ slightly exceeds $4 \pi^{3}\left(n^{2}-1\right)$.

We can now use (6.1) to determine $\theta(\xi)$, namely

$$
\begin{aligned}
\theta & \sim 2 \pi \xi+\frac{2 \delta \pi\left(n^{2}-1\right) a_{1}}{n} \sin (2 n \pi \xi) \\
& =2 \pi \xi-\sqrt{\frac{2\left(p-4 \pi^{3}\left(n^{2}-1\right)\right)}{3 \pi^{3}\left(n^{2}-1\right)}} \sin (2 n \pi \xi) .
\end{aligned}
$$

For each value of $n$, we wish to determine the value of $p$ such that the profile most closely resembles an $n$-sided polygon. This is essentially an aesthetic decision, and we will choose to make the curvature equal to zero at the centre of each edge. Thus we set $\theta^{\prime}(0)=0$ and deduce from (6.12) the condition

$$
p=4 \pi^{3}\left(n^{2}-1\right)+\frac{3 \pi^{3}\left(n^{2}-1\right)}{2 n^{2}}=\frac{\left(8 n^{2}+3\right)\left(n^{2}-1\right) \pi^{3}}{2 n^{2}} .
$$

We see from (6.13) that the approximation for $p$ is asymptotic only in the limit $n \rightarrow \infty$. 

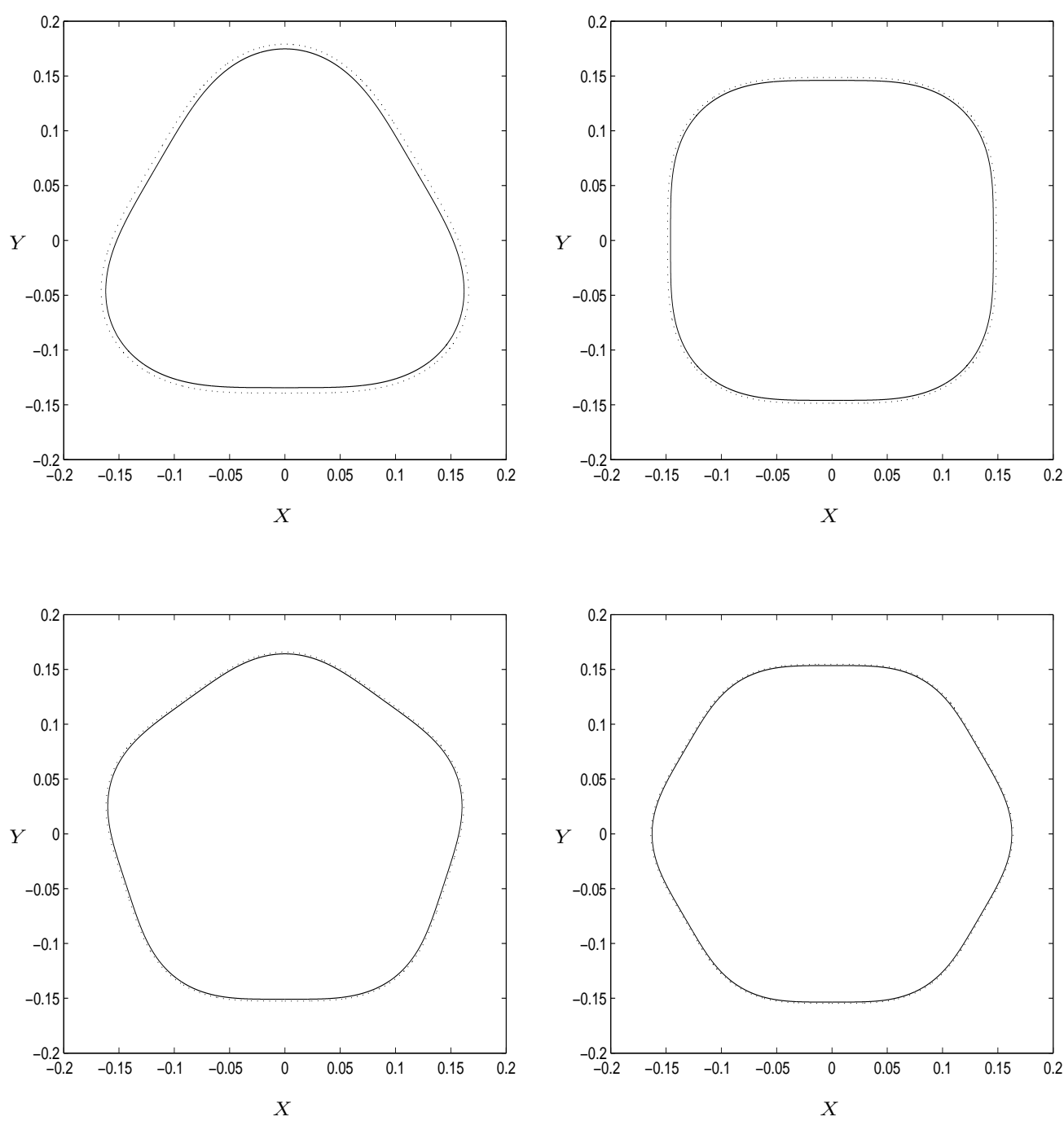

FiguRE 15. Approximately polygonal steady shapes with $n=3,4,5,6$. Solid lines show nonlinear numerical solutions, dashed lines show the weakly nonlinear approximation.

However, figure 15 shows remarkably good agreement between the weakly nonlinear approximation (6.12), with $p$ given by (6.13), and fully nonlinear numerical solutions. The graphs are very close even when $n=3$, and converge rapidly as $n$ increases. This clearly demonstrates the existence of steady solutions that are a good representation of the polygonal shapes desired and, in addition, that these shapes are well approximated by the weakly nonlinear theory. We note that the condition $\theta^{\prime}(0)=0$ simplifies the nonlinear analysis of $\S 5$ since it imposes the condition $\beta=1 / 2 \nu$. However, it seems unlikely that we could arrange in practice for the pressure to vary in a prescribed fashion such as (5.1). If $\mathcal{P}$ does not satisfy (5.1), then we must abandon the assumption that the shape of the viscida is conserved. However, the surprisingly good approximation afforded by the weakly nonlinear theory motivates us to try the same approach for unsteady solutions. 


\subsection{Unsteady solutions}

Now let us consider unsteady solutions of (3.37) which, in terms of $R$, reads

$$
\frac{\partial}{\partial \tau}\left((1+\tau / 2) \frac{1-R_{\xi}^{2}-R R_{\xi \xi}}{R \sqrt{1-R_{\xi}^{2}}}\right)=\frac{p}{2} R^{2}+C,
$$

where $p$ is still defined by (5.1) but is no longer assumed to be constant. To facilitate the weakly nonlinear analysis, we introduce the slow timescale

$$
\hat{\tau}=2 \delta^{2} \ln (1+\tau / 2)
$$

so that (6.14) becomes

$$
\frac{1-R_{\xi}^{2}-R R_{\xi \xi}}{R \sqrt{1-R_{\xi}^{2}}}+2 \delta^{2} \frac{\partial}{\partial \hat{\tau}}\left(\frac{1-R_{\xi}^{2}-R R_{\xi \xi}}{R \sqrt{1-R_{\xi}^{2}}}\right)=p R^{2}+2 C .
$$

Now we can follow a perturbative approach analogous to that used in $\S 6.1$, but letting the dependent variables depend on $\hat{\tau}$ as follows:

$$
\begin{aligned}
p(\hat{\tau}) & =4 \pi^{3}\left(n^{2}-1\right)+\delta^{2} p^{(2)}(\hat{\tau})+\cdots, \\
R(\xi, \hat{\tau}) & \sim 1 / 2 \pi+\delta a_{1}(\hat{\tau}) \cos (2 n \pi \xi)+\delta^{2} R^{(2)}(\xi, \hat{\tau})+\cdots \\
C(\hat{\tau}) & \sim\left(3-n^{2}\right) \pi / 2+\delta^{2} C^{(2)}(\hat{\tau})+\cdots .
\end{aligned}
$$

Now the solvability condition at order $\delta^{3}$ yields an ordinary differential equation for $a_{1}$, namely

$$
\frac{\mathrm{d} a_{1}}{\mathrm{~d} \hat{\tau}}=\frac{a_{1}}{8 \pi^{3} n^{2}\left(n^{2}-1\right)}\left(n^{2} p^{(2)}-6 \pi^{5}\left(n^{2}-1\right)^{3} a_{1}^{2}\right)
$$

Notice that this reduces to $(6.10)$ for steady solutions with $p^{(2)} \equiv 1$. In terms of the original unscaled variables, (6.20) becomes

$$
\begin{aligned}
(1+\tau / 2) \frac{\mathrm{d} a}{\mathrm{~d} \tau}=\frac{a}{8 n^{2}\left(n^{2}-1\right) \pi^{3}}\left(-\frac{3 n^{2} \mathcal{P}}{(1+\tau / 2)^{5}}\right. & \begin{array}{r}
\left.-4 \pi^{3} n^{2}\left(n^{2}-1\right)-6 \pi^{5}\left(n^{2}-1\right)^{3} a^{2}\right)
\end{array}
\end{aligned}
$$

where $a(\tau)=\delta a_{1}$.

Equation (6.21) describes weakly nonlinear time-dependent solutions close to the $n$ lobed bifurcation. The most physically realistic scenario occurs when $\mathcal{P}$ is constant, in which case (6.21) may be solved explicitly in the form

$$
a= \pm \frac{(-\mathcal{P})^{1 / 5} n \exp \left(3 \mathcal{P} / 20\left(n^{2}-1\right) \pi^{3}(1+\tau / 2)^{5}\right)}{(1+\tau / 2) \sqrt{K+\frac{2^{2 / 5} 3^{3 / 5}\left(n^{2}-1\right)^{12 / 5} \pi^{16 / 5}}{5^{3 / 5}} \Gamma\left(\frac{2}{5}, \frac{-3 \mathcal{P}}{10\left(n^{2}-1\right) \pi^{3}(1+\tau / 2)^{5}}\right)}},
$$

where $\Gamma$ is the incomplete Gamma function (Gradshteyn \& Ryzhik 1994, section 8.35) and the constant $K$ is set by the initial condition. For the sake of argument, let us choose 


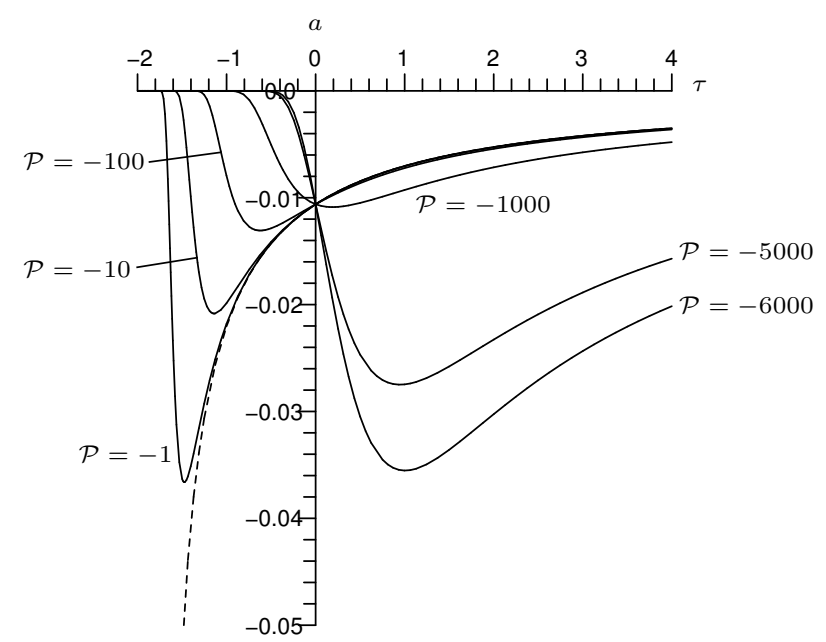

FigURE 16. Plot of weakly nonlinear amplitude $a$ versus $\tau$ for various values of $\mathcal{P}$ when $n=4$. The dashed line shows the limiting solution as $\mathcal{P} \rightarrow 0$.

$\theta_{\xi}(0,0)=0$, so that the profile is initially close to polygonal. This leads to

$$
\begin{aligned}
K=4 n^{2} 3^{2 / 5}\left(n^{2}-1\right)^{2} \pi^{2}(-\mathcal{P})^{2 / 5} \exp \left(\frac{3 \mathcal{P}}{10\left(n^{2}-1\right) \pi^{3}}\right) & \\
& -\frac{2^{2 / 5} 3^{3 / 5}\left(n^{2}-1\right)^{12 / 5} \pi^{16 / 5}}{5^{3 / 5}} \Gamma\left(\frac{2}{5}, \frac{-3 \mathcal{P}}{10\left(n^{2}-1\right) \pi^{3}}\right)
\end{aligned}
$$

and we choose the negative root for $a$ in (6.22).

In figure 16 we plot the solution (6.22) versus $\tau$ for $n=4$ and various values of $\mathcal{P}$. We observe that typically the amplitude increases initially with $\tau$, before decreasing again, tending to zero at large times as the profile approaches a circle. Consequently, for any given $\mathcal{P}$ there will be a particular time, either positive or negative, at which the viscida returns to its initial, roughly polygonal, shape. The limiting case as $\mathcal{P} \rightarrow 0$, when (6.22) reduces to

$$
a \sim-\frac{n \sqrt{2}}{\pi\left(n^{2}-1\right) \sqrt{\left(8 n^{2}+3\right)(1+\tau / 2)^{2}-3}},
$$

is also shown as a dashed curve.

The solution (6.22) thus supports the graphical observations of $\S 4.2$, where the profiles were observed to return approximately to their original shapes. However, we must acknowledge that the weakly nonlinear analysis is formally valid only when $p$ is close to one of the bifurcation values, which is not uniformly true when $\mathcal{P}$ is held fixed. In figure 17 , we compare the time-dependent weakly nonlinear solution with the fully nonlinear numerical solution corresponding to an initial approximately square shape. The agreement is remarkably good, even though the asymptotic assumptions underlying the weakly nonlinear analysis are clearly violated in this case. Moreover, we observe that the profile buckles inwards before approximately recovering its initial shape (when $\tau \approx-1.4$ ) and finally tending towards a circle. 

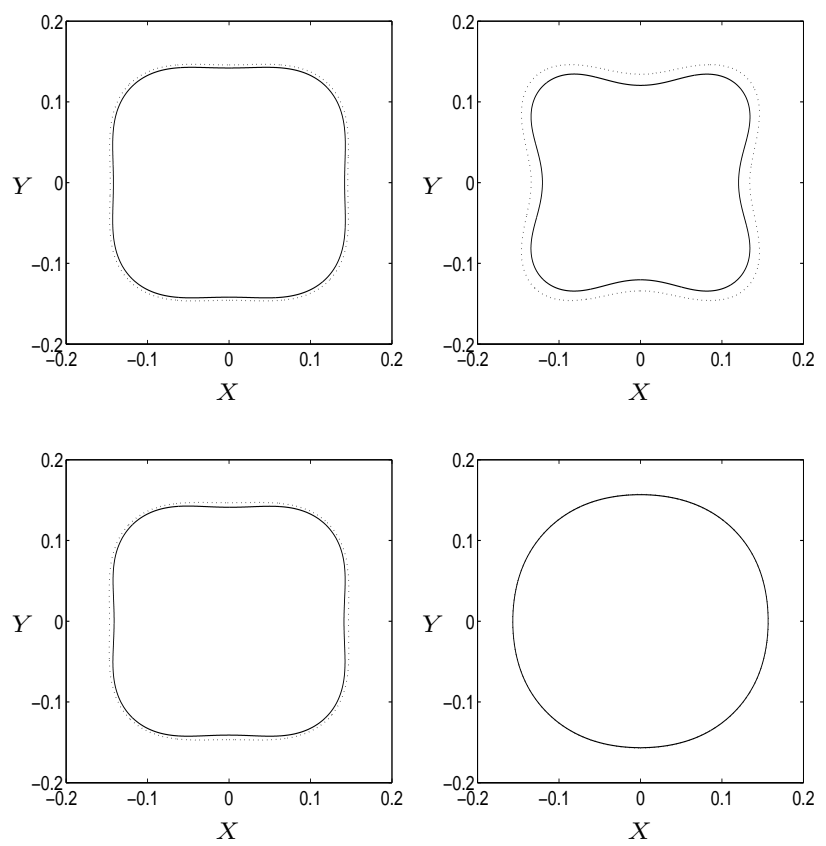

FIGURE 17. Comparison of weakly nonlinear solution (dashed) and fully nonlinear solution (solid) for $\mathcal{P}=-10$ and $\tau=0,-1.2,-1.4,-1.5$.

\section{Conclusions}

This paper concerns two-dimensional Stokes flow driven by surface tension in an annular domain, and it is worth noting that exact solutions to this problem exist (see Crowdy \& Tanveer 1998; Crowdy 2003; Richardson 2000). However, the theory of such solutions is difficult to apply to arbitrary shapes and cannot be used when non-zero pressures are applied to the free boundaries. We therefore chose instead to assume that the domain has a thickness much smaller than its circumference, so it may be modelled as a thin viscida. This allowed us to derive a simplified asymptotic model, generalizing that of Buckmaster \& Nachman (1978) and comprising an intrinsic equation governing the evolution of the centre-line of the viscida. It would be worthwhile and interesting to use exact solution techniques to test the validity of our asymptotic simplifications.

In the case where there is no internal pressurization and the profile possesses rotational symmetry, our model admits an exact analytic solution. This allows us to observe how the tube evolves towards a circular profile as time increases and also to solve explicitly the inverse problem of determining the initial shape corresponding to a desired final shape. The inverse problem is surprisingly well posed, although the solutions do eventually break down by self-intersection, suggesting that some final shapes may be impossible to realize. The full two-dimensional surface-tension-driven Stokes flow problem is undoubtedly ill posed when solved backwards in time, so our asymptotic simplification has the fortuitous side-effect of eliminating unstable high-wavenumber disturbances.

When internal pressurization is included, analytic solution is no longer possible but our simplified model is readily solved numerically. We found that a positive applied pressure accelerates the evolution towards a circle, while suction delays it. For negative time, the roles of positive and negative pressures are reversed: applying a positive pressure when time runs backwards has a qualitatively similar effect to a negative pressure when 
time increases. Of particular interest is the fact that, if we choose the applied pressure appropriately, its competition with surface tension may serve to hold the initial profile approximately constant in time, so that the only noticeable change is in its overall size. Alternatively, it may be possible for the evolution to be dominated initially by the applied pressure and subsequently by surface tension, so that the profile returns approximately to its initial shape after some transient.

To aid the understanding of these observations, we considered the existence of steady solutions, namely profiles which maintain their shape while shrinking uniformly with time. We found that these exist provided the applied pressure is proportional to $(1+\tau / 2)^{5}$, in which case the equation for the viscida is equivalent to that for an elastica under a uniform normal pressure. We noticed that some of these steady solutions resemble quite closely the approximately polygonal shapes that we are particularly interested in producing, and that these shapes may be regarded as perturbations to a circle. This allowed us to derive a simplified weakly nonlinear model that gives surprisingly good agreement with the fully nonlinear model, even when it is far from being asymptotically valid. This analysis confirms that we can arrange for the initial profile to be recovered after a given interval by applying an appropriate internal pressure.

This theory is applicable for a thin viscida whose thickness remains everywhere much smaller than its radius of curvature. If this condition is violated our theory is no longer valid and analysis of the full two-dimensional Stokes equations is required. However, we found that increasing the curvature so that the viscida profile approaches a configuration containing corner regions did not generate any adverse effects in the global behaviour of the viscida evolution. Consequently, we concluded that the system may be described by our analysis for the majority of the viscida, except in small inner regions near any corners where the curvature is large. To determine the evolution in these regions requires consideration of the full two-dimensional Stokes equations, but any two-dimensional effects are confined to a neighbourhood of the corners and do not influence the global sheet evolution.

The original motivation to consider this problem arose from the concept of manufacturing capillary tubing with a specific cross-sectional shape, where the aim is to solve the inverse problem of determining the die shape required to achieve a given final cross-section. It is demonstrated in Cummings \& Howell (1999) that the shape of the cross-section of a simply-connected slowly varying non-axisymmetric fibre satisfies a two-dimensional time-dependent Stokes flow problem, when expressed in suitable scaled Lagrangian coordinates. In a subsequent paper we will show that this result may be generalized for hollow temperature-dependent fibres, so that the evolution of the cross-section with axial position may be related to the time evolution of a constant-viscosity annular viscida. The results of this paper thus allow us to solve the inverse die problem for a slowly varying three-dimensional tubing. In particular, the results of $\S 6$ indicate that, by using a die shaped as shown in figure 15 and applying an appropriate internal pressurization, the final tube cross-section can be manufactured as an identical scaled copy of the initial die. By varying the applied pressure, we can vary the time taken for the initial shape to be recovered and hence the size of the cross-section when it achieves the desired shape. This result in principle allows us to use a single die to produce tubes with identical cross-section shapes but differing sizes.

This research was supported by a studentship from Oxford's EPSRC-funded doctoral training account. The authors gratefully acknowledge helpful discussions with Dr H. J. J. Gramberg, Professor E. J. Hinch, Dr J. R. Lister and Dr J. R. Ockendon. We 
were introduced to this problem by U. Lange from Schott AG, who gave us invaluable insight into the practical issues involved.

\section{REFERENCES}

Buckmaster, J. D. \& Nachman, A. 1978 The buckling and stretching of a viscida II. Effects of surface tension. Quart. J. Mech. Appl. Math. 31, 157-168.

Buckmaster, J. D., Nachman, A. \& Ting, L. 1975 The buckling and stretching of a viscida. J. Fluid Mech. 69, 1-20.

Crowdy, D. 2003 Viscous sintering of unimodal and bimodal cylindrical packings with shrinking pores. Eur. J. Appl. Math. 14, 421-445.

Crowdy, D. \& Tanveer, S. 1998 A theory of exact solutions for annular viscous blobs. J. Nonlinear Sci. 8, 375-400.

Cummings, L. J. \& Howell, P. D. 1999 On the evolution of non-axisymmetric viscous fibres with surface tension, inertia and gravity. J. Fluid Mech. 389, 361-389.

Fitt, A. D., Furusawa, K., Monro, T. M., Please, C. P. \& Richardson, D. A. 2002 The mathematical modelling of capillary drawing for holey fibre manufacture. J. Eng. Math. 43, 201-227.

Fitt, A. D., Furusawa K., Monro, T. M. \& Please, C. P. 2001 Modeling the fabrication of hollow fibres: Capillary drawing. J. Lightw. Technol. 19, 1924-1931.

van De Fliert, B. W., Howell, P. D. \& OCKendon, J. R. 1995 Pressure-driven flow of a thin viscous sheet. J. Fluid Mech. 292, 359-376.

Gradshteyn, I. S. \& Ryzhik, I. M. 1994 Table of Integrals, Series, and Products. Fifth Edition.. Academic Press.

Graham, S. J. 1987 Mathematical modelling of glass flow in container manufacture. PhD thesis, University of Sheffield.

Kreyszig, E. 1959 Differential Geometry. University of Toronto Press (reprinted Dover, 1991).

Love, A. E. H. 1927 A Treatise on the Mathematical Theory of Elasticity. Cambridge University Press (reprinted Dover, 1944).

Pfaender, H. G. 1996 Schott Guide to Glass. Chapman and Hall, London.

Ribe, N. M. 2001 Bending and stretching of thin viscous sheets. J. Fluid Mech. 433, 135-160.

Richardson, S. 2000 Plane Stokes flows with time-dependent free boundaries in which the fluid occupies a doubly-connected region. Euro. J. Appl. Math. 11, 249-269.

Sivko, A. P. 1976 The production of glass tubes using the Vello method. Glass and Ceramics 33, 728-730.

Uhlmann, D. R. \& Kreidu, N. J. 1984 Glass. Science and Technology. Volume 2. Processing I. Academic Press.

Wu, C. Y., Somervell, A. R. D. \& Barnes, T. H. 1998 Direct image transmission through a multi-mode square optical fiber. Optics Communications 157, 17-22.

Wu, C. Y., Somervell, A. R. D., Haskell, T. G. \& Barnes, T. H. 2000 Optical sine transformation and image transmission by using square optical waveguide. Optics Communications 175, 27-32. 